\title{
Extremal Functions of the Singular Moser-Trudinger Inequality Involving the Eigenvalue
}

\author{
ZHOU Changliang and ZHOU Chunqin* \\ School of Mathematical Sciences, Shanghai Jiao Tong University, Shanghai 200240, \\ China.
}

Received 8 September 2017; Accepted 18 January 2018

\begin{abstract}
In this paper, we derive the singular Moser-Trudinger inequality which involves the first eigenvalue and several singular points, and further prove the existence of the extremal functions for the relative Moser-Trudinger functional. Since the problems involve more complicated norm and multiple singular points, not only we can't use the symmetrization to deal with a one-dimensional inequality, but also the processes of the blow-up analysis become more delicate. In particular, the new inequality is more general than that of $[1,2]$.
\end{abstract}

AMS Subject Classifications: 46E35

Chinese Library Classifications: O175.25

Key Words: Singular Moser-Trudinger inequlaity; existence of extremal functions; blow up analysis.

\section{Introduction}

Let $\Omega \subset \mathbb{R}^{2}$ be a smooth bounded domain. The famous Moser-Trudinger inequality [3-5] says that

$$
\sup _{u \in W_{0}^{1,2}(\Omega),\|\nabla u\|_{L^{2}(\Omega) \leq 1}} \int_{\Omega} e^{\sigma|u|^{2}} \mathrm{~d} x<+\infty
$$

for any $\sigma \leq 4 \pi$. Moreover, for any fixed $u \in W_{0}^{1,2}(\Omega)$, it also holds that

$$
\int_{\Omega} e^{\sigma|u|^{2}} \mathrm{~d} x<+\infty
$$

*Corresponding author. Email addresses: zhzh1800130@sjtu.edu.cn (C.L. Zhou), cqzhou@sjtu.edu.cn (C.Q. Zhou) 
for any $\sigma>0$. In particular, the constant $\sigma=4 \pi$ is optimal in (1.1), which implies that, for any $\sigma>4 \pi$, the inequality (1.1) is invalid and there exists a sequence of $\left\{u_{\epsilon}\right\}$ in $W_{0}^{1,2}(\Omega)$ and $\left\|\nabla u_{\epsilon}\right\|_{L^{2}(\Omega)}=1$ such that

$$
\int_{\Omega} e^{\sigma\left|u_{\epsilon}\right|^{2}} \mathrm{~d} x \rightarrow \infty \quad \text { as } \epsilon \rightarrow 0
$$

Moser-Trudinger inequality (1.1), as a limit case of the Sobolev embedding, plays an important role in two-dimensional analytic and geometric problems. The further interesting subject is the existence of extremal functions to (1.1). By using the blow-up method Carleson and Chang [6] showed that the supremum is actually attained if $\Omega$ is a ball. Flucher [7] generalized this result to arbitrary bounded domains in $\mathbb{R}^{2}$. See also Adimurthi-Tintarev [8], Malchiodi-Martinazzi [9] and Mancini-Sandeep [10] and the references in these papers for recent developments on this subject.

This inequality was generalized in many ways. One kind of generalization of (1.1) is the so-called singular Moser-Trudinger inequality, which was originally established by Adimurthi-Sandeep [11]. They proved that

$$
\sup _{u \in W_{0}^{1,2}(\Omega),\|\nabla u\|_{L^{2}(\Omega)} \leq 1} \int_{\Omega} \frac{e^{s u^{2}}-1}{|x|^{2 t}} \mathrm{~d} x<+\infty,
$$

for $s \in(0,4 \pi(1-t))$ and $t \in[0,1)$. Further, Csató-Roy [12] proved that the supremum is attained for this singular Moser-Trudinger embedding.

For the case of several singular points, Iula-Mancini [1] proved that the supremum

$$
\sup _{u \in W_{0}^{1,2}(\Omega), \int_{\Omega}|\nabla u|^{2} \mathrm{~d} x \leq 1} \int_{\Omega} V(x) e^{4 \pi(1+\alpha)\left(1+\left.\lambda|| u\right|_{L^{\prime}(\Omega)} ^{2}\right)} \mathrm{d} x
$$

is finite and is attained for $\lambda \in\left[0, \lambda_{q}(\Omega)\right)$. Here

$$
\lambda_{q}(\Omega)=\inf _{u \in W_{0}^{1,2}(\Omega), \int_{\Omega}|\nabla u|^{2} \mathrm{~d} x \leq 1} \frac{\int_{\Omega}|\nabla u|^{2} \mathrm{~d} x}{\|u\|_{L^{q}(\Omega)}^{2}}
$$

for $q>1$, and

$$
V(x)=K(x) \prod_{i=1}^{m}\left|x-p_{i}\right|^{2 \alpha_{i}}
$$

where $K(x)>0, K(x) \in C^{0}(\bar{\Omega}) ; p_{1}, p_{2}, \cdots, p_{m}$ are the different points in $\Omega$; and $\alpha_{i} \in(-1,+\infty)$, $\alpha_{i} \notin \mathbb{Z}$ such that

$$
\alpha=\min _{1 \leq i \leq m}\left\{\alpha_{i}\right\} \quad \text { and } \alpha \in(-1,0)
$$


Now we describe another kind of generalization of (1.1). Tintarev [13] introduced the first eigenvalue

$$
\lambda_{1}(\Omega)=\inf _{u \in W_{0}^{1,2}(\Omega), u \neq 0} \frac{\int_{\Omega}|\nabla u|^{2} \mathrm{~d} x}{\int_{\Omega} u^{2} \mathrm{~d} x}
$$

to the Moser-Trudinger inequality. Instead of the usual sobolev norm, he take an equivalent norm of each $u$ in $W_{0}^{1,2}(\Omega)$

$$
\|u\|_{1, \beta}=\left(\int_{\Omega}|\nabla u|^{2} \mathrm{~d} x-\beta \int_{\Omega} u^{2} \mathrm{~d} x\right)^{\frac{1}{2}},
$$

where $\beta<\lambda_{1}(\Omega)$. Then he proved that the supremum

$$
\sup _{u \in W_{0}^{1,2}(\Omega),\|u\|_{1, \beta} \leq 1} \int_{\Omega} e^{4 \pi u^{2}} \mathrm{~d} x
$$

is finite.

Later, Yang-Zhu [2] extended Tintarev'result to Moser-Trudinger inequalities with a singular point, i.e. they prove the supremum

$$
\sup _{u \in W_{0}^{1,2}(\Omega),\|u\|_{1, \beta} \leq 1} \int_{\Omega} \frac{e^{4 \pi(1-\alpha)} u^{2}}{|x|^{2 \alpha}} \mathrm{d} x
$$

is finite and is attained for $\beta \in\left[0, \lambda_{1}(\Omega)\right)$ and $\alpha \in(0,1)$.

For more generalizations of the classical Moser-Trudinger inequality (1.1), one can see for instance $[12,14-22]$ and the reference therein.

In this paper, we want to introduce the equivalent norm (1.5) and the multiple singular points to the Moser-Trudinger inequality at the same time. The new inequality is more general than that of $([1,2])$. We also show the existence of the extremal functions for such stronger inequalities. Our main results are stated as following:

Theorem 1.1. Let $\Omega \subset \mathbb{R}^{2}$ be a smooth bounded domain and $V(x)$ be as in (1.3). Let $\alpha \in(-1,0)$ be fixed and $\lambda_{1}(\Omega)$ be defined as in (1.4). Then for any $\beta<\lambda_{1}(\Omega)$, we have

$$
\sup _{u \in W_{0}^{1,2}(\Omega),\|u\|_{1, \beta} \leq 1} \int_{\Omega} V(x) e^{4 \pi(1+\alpha) u^{2}} \mathrm{~d} x<+\infty,
$$

where $\|u\|_{1, \beta}$ is defined as in (1.5).

Theorem 1.2. Under the assumption of Theorem 1.1, there exists some function $u_{0} \in W_{0}^{1,2}(\Omega) \cap$ $C^{0}(\bar{\Omega})$ with $\left\|u_{0}\right\|_{1, \beta}=1$ satisfying

$$
\int_{\Omega} V(x) e^{4 \pi(1+\alpha) u_{0}^{2}} \mathrm{~d} x=\sup _{u \in W_{0}^{1,2}(\Omega),\|u\|_{1, \beta} \leq 1} \int_{\Omega} V(x) e^{4 \pi(1+\alpha) u^{2}} \mathrm{~d} x
$$

for any $\beta<\lambda_{1}(\Omega)$. 
For the proof of our results, we use an important tool in geometric analysis, the blowup analysis. Since the problems involve more complicated norm and multiple singular points, not only we can't use the symmetrization to deal with a one-dimensional inequality, but also the steps of the blow-up analysis become more delicate. Because of the presence of several singularities, it is difficult to identify the number of the blow up point and to locate the position of the blow up point when the maximizing sequence blows up. Actually, in the Section 3, we illustrate the processes of identifying the number of the blow up point and locating the position of the blow up point by combining the classification results of Chen-Li [23] and that of Prajapat-Tarantello [24]. We should mention that we finally prove that the only blow up point is the singular point with the least power $\alpha$ and consequently get the desired bubble.

\section{Maximizers for subcritical-Moser-Trudinger functional}

In this section, we will show the existence of the maximizers for Moser-Trudinger functional in the subcritical case. Let us start with two useful Lemmas. The first Lemma is an embedding Lemma of Orlicz type, i.e.

Lemma 2.1. Let $V(x)$ be as in (1.3) and $u \in W_{0}^{1,2}(\Omega)$. For any $p>0$, and any $\gamma>0$ such that $-1<\alpha \gamma<0$, there holds

$$
\int_{\Omega} V^{\gamma}(x) e^{4 \pi p(1+\alpha \gamma) u^{2}} \mathrm{~d} x<+\infty .
$$

Proof. For any fixed $p>0$, we take $q>1$ such that $-2<2 \alpha \gamma q<0$. By the Hölder inequality, we have

$$
\int_{\Omega} V^{\gamma}(x) e^{4 \pi p(1+\alpha \gamma) u^{2}} \mathrm{~d} x \leq\left(\int_{\Omega}(V(x))^{\gamma q} \mathrm{~d} x\right)^{\frac{1}{q}} \cdot\left(\int_{\Omega} e^{4 \pi q^{*} p(1+\alpha \gamma) u^{2}} \mathrm{~d} x\right)^{\frac{1}{q^{*}}}<+\infty,
$$

where $q^{*}=\frac{q}{q-1}$.

The other useful Lemma is the following, which is obviously obtained from (1.2).

Lemma 2.2. For any $\beta>0$ satisfying $-1<\alpha \beta<0$, there holds

$$
\sup _{u \in W_{0}^{1,2}(\Omega),\|\nabla u\|_{L^{2}(\Omega)} \leq 1} \int_{\Omega} V^{\beta}(x) e^{4 \pi(1+\alpha \beta) u^{2}} \mathrm{~d} x<+\infty .
$$

Proposition 2.1. For any $\epsilon \in(0,1+\alpha)$, there exist some $u_{\epsilon} \in W_{0}^{1,2}(\Omega) \cap C^{0}(\bar{\Omega})$ satisfying $\left\|u_{\epsilon}\right\|_{1, \beta}=$ 1 and

$$
\int_{\Omega} V(x) e^{4 \pi(1+\alpha-\epsilon) u_{\epsilon}^{2}} \mathrm{~d} x=\sup _{u \in W_{0}^{1,2}(\Omega),\|u\|_{1, \beta} \leq 1} \int_{\Omega} V(x) e^{4 \pi(1+\alpha-\epsilon) u^{2}} \mathrm{~d} x .
$$


In particular, $u_{\epsilon}$ satisfies the following Dirichlet problem

$$
\begin{cases}-\Delta u_{\epsilon}-\beta u_{\epsilon}=\frac{u_{\epsilon}}{\lambda_{\epsilon}} V(x) e^{4 \pi(1+\alpha-\epsilon) u_{\epsilon}^{2}} & \text { in } \Omega, \\ u_{\epsilon}=0 & \text { on } \frac{\partial \Omega}{\Omega} \\ u_{\epsilon} \geq 0 & \text { on } \bar{\Omega}\end{cases}
$$

in the distributional sense, where $\lambda_{\epsilon}=\int_{\Omega} u_{\epsilon}^{2} V(x) e^{4 \pi(1+\alpha-\epsilon)} u_{\epsilon}^{2} \mathrm{~d} x$.

Proof. For any fixed $\epsilon \in(0,1+\alpha)$, we let $u_{\epsilon, j}$ be a maximizing sequence in $W_{0}^{1,2}(\Omega)$ with $\left\|u_{\epsilon, j}\right\|_{1, \beta} \leq 1$. Since $\beta<\lambda_{1}(\Omega)$, we have

$$
\left(1-\frac{\beta}{\lambda_{1}(\Omega)}\right) \int_{\Omega}\left|\nabla u_{\epsilon, j}\right|^{2} \mathrm{~d} x \leq \int_{\Omega}\left|\nabla u_{\epsilon, j}\right|^{2} \mathrm{~d} x-\beta \int_{\Omega} u_{\epsilon, j}^{2} \mathrm{~d} x \leq 1,
$$

which implies that $u_{\epsilon, j}$ is bounded in $W_{0}^{1,2}(\Omega)$. Hence their exists some $u_{\epsilon, j} \in W_{0}^{1,2}(\Omega)$ such that up to a subsequence,

$$
\begin{aligned}
& u_{\epsilon, j} \rightarrow u_{\epsilon} \text { weakly in } W_{0}^{1,2}(\Omega), \\
& u_{\epsilon, j} \rightarrow u_{\epsilon} \text { strongly in } L^{p}(\Omega), \text { for any } p \geq 1, \\
& u_{\epsilon, j} \rightarrow u_{\epsilon} \text { a.e. } \Omega .
\end{aligned}
$$

For any $1<p<-\frac{1}{\alpha}, \delta>0, s>1$, and $s^{*}=\frac{s}{s-1}$, by the Hölder inequality and the inequality

$$
u_{\epsilon, j}^{2} \leq(1+\delta)\left(u_{\epsilon, j}-u_{\epsilon}\right)^{2}+\left(1+\frac{1}{4 \delta}\right) u_{\epsilon}^{2}
$$

we have

$$
\begin{aligned}
& \int_{\Omega} V^{p}(x) e^{4 \pi(1+\alpha-\epsilon) p u_{\epsilon, j}^{2}} \mathrm{~d} x \\
\leq & \int_{\Omega} V^{p}(x) e^{4 \pi(1+\alpha-\epsilon)(1+\delta) p\left(u_{\epsilon, j}-u_{\epsilon}\right)^{2}+4 \pi(1+\alpha-\epsilon) p\left(1+\frac{1}{4 \delta}\right) u_{\epsilon}^{2}} \mathrm{~d} x \\
\leq & \left(\int_{\Omega} V^{p}(x) e^{4 \pi(1+\alpha-\epsilon)(1+\delta) p s\left(u_{\epsilon, j}-u_{\epsilon}\right)^{2}}\right)^{\frac{1}{s}}\left(\int_{\Omega} V^{p}(x) e^{4 \pi(1+\alpha-\epsilon) p s^{*}\left(1+\frac{1}{4 \delta}\right) u_{\epsilon}^{2}} \mathrm{~d} x\right)^{\frac{1}{s^{*}}} .
\end{aligned}
$$

Choose $p, 1+\delta, s$ sufficiently close to 1 such that

$$
(-\alpha p)+(1+\alpha-\epsilon) p s(1+\delta) \leq 1 .
$$

Clearly we have that

$$
0 \leq \int_{\Omega}\left|\nabla u_{\epsilon}\right|^{2} \mathrm{~d} x-\beta \int_{\Omega} u_{\epsilon}^{2} \mathrm{~d} x \leq \liminf _{j \rightarrow+\infty}\left(\int_{\Omega}\left|\nabla u_{\epsilon, j}\right|^{2} \mathrm{~d} x-\beta \int_{\Omega} u_{\epsilon, j}^{2} \mathrm{~d} x\right) \leq 1,
$$


and that

$$
\begin{aligned}
\int_{\Omega}\left|\nabla u_{\epsilon, j}-\nabla u_{\epsilon}\right|^{2} \mathrm{~d} x & =\int_{\Omega}\left|\nabla u_{\epsilon, j}\right|^{2} \mathrm{~d} x-\int_{\Omega}\left|\nabla u_{\epsilon}\right|^{2} \mathrm{~d} x+o_{j}(1) \\
& \leq 1-\int_{\Omega}\left|\nabla u_{\epsilon}\right|^{2} \mathrm{~d} x+\beta \int_{\Omega} u_{\epsilon}^{2} \mathrm{~d} x+o_{j}(1) .
\end{aligned}
$$

Combining (2.6) and (2.7), we conclude limsup $\operatorname{sit\infty }_{j \rightarrow \infty}\left|\nabla u_{\epsilon, j}-\nabla u_{\epsilon}\right|^{2} \mathrm{~d} x \leq 1$. Inserting (2.6) and (2.7) into (2.4), by Lemma 2.2 and Lemma 2.1, we have $V(x) e^{4 \pi(1+\alpha-\epsilon) u_{\epsilon, j}^{2}}$ is uniformly bounded in $L^{q}(\Omega)$ for some $q>1$.

Since

$$
\begin{aligned}
& \left|e^{4 \pi(1+\alpha-\epsilon) u_{\epsilon, j}^{2}}-e^{4 \pi(1+\alpha-\epsilon) u_{\epsilon}^{2}}\right| \\
\leq & \left.4 \pi(1+\alpha-\epsilon)\left(e^{4 \pi(1+\alpha-\epsilon) u_{\epsilon, j}^{2}}+e^{4 \pi(1+\alpha-\epsilon)}\right) u_{\epsilon}^{2}\right)\left|u_{\epsilon, j}^{2}-u_{\epsilon}^{2}\right|,
\end{aligned}
$$

and $u_{\epsilon, j} \rightarrow u_{\epsilon}$ strongly in $L^{p}(\Omega)$ for any $p \geq 1$ as $j \rightarrow+\infty$, we conclude that

$$
\limsup _{j \rightarrow+\infty} \int_{\Omega} V(x) e^{4 \pi(1+\alpha-\epsilon) u_{\epsilon, j}^{2}} \mathrm{~d} x=\int_{\Omega} V(x) e^{4 \pi(1+\alpha-\epsilon) u_{\epsilon}^{2}} \mathrm{~d} x
$$

Thus we have that $u_{\epsilon}$ attains the supremum. Clearly $u_{\epsilon} \not \equiv 0$. If we suppose that $\left\|u_{\epsilon}\right\|_{1, \beta}<$ 1. It follows that

$$
\int_{\Omega} V(x) e^{4 \pi(1+\alpha-\epsilon) u_{\epsilon}^{2}} \mathrm{~d} x<\int_{\Omega} V(x) e^{4 \pi(1+\alpha-\epsilon)\left(\frac{u_{\epsilon}}{\left\|u_{\epsilon}\right\|_{1, \beta}}\right)^{2}} \mathrm{~d} x,
$$

which is a contradiction. Hence we have $\left\|u_{\epsilon}\right\|_{1, \beta}=1$. A straightforward calculation shows that $u_{\epsilon}$ satisfies the Euler-Lagrange equation (2.3) in the distributional sense.

Moreover, using the Hölder inequality and Lemma 2.1, for $p>1, r>1, q>1$ such that $-1<p q \alpha<0$ and $\operatorname{pr}(1+\alpha) \leq 1+\alpha p$, we can have that

$$
\begin{aligned}
& \int_{\Omega}(V(x))^{p}\left(u_{\epsilon}\right)^{p} e^{4 \pi p(1+\alpha-\epsilon) u_{\epsilon}^{2}} \mathrm{~d} x \\
\leq & \left(\int_{\Omega}(V(x))^{p}\left(u_{\epsilon}\right)^{r^{*} p} \mathrm{~d} x\right)^{\frac{1}{r^{*}}}\left(\int_{\Omega}(V(x))^{p} e^{4 \pi p r(1+\alpha-\epsilon) u_{\epsilon}^{2}} \mathrm{~d} x\right)^{\frac{1}{r}} \\
\leq & \left(\int_{\Omega}(V(x))^{p q} \mathrm{~d} x\right)^{\frac{1}{q^{*}}}\left(\int_{\Omega} u_{\epsilon}^{r^{*} p q^{*}} \mathrm{~d} x\right)^{\frac{1}{r^{*} q^{*}}}\left(\int_{\Omega}(V(x))^{p} e^{4 \pi p r(1+\alpha-\epsilon) u_{\epsilon}^{2}} \mathrm{~d} x\right)^{\frac{1}{r}}<+\infty .
\end{aligned}
$$

So $\frac{1}{\lambda_{\epsilon}} V(x) u_{\epsilon} e^{4 \pi(1+\alpha-\epsilon)} u_{\epsilon}^{2}$ is bounded in $L^{p}(\Omega)$ for some $p>1$. By the standard elliptic estimation, we have that $u_{\epsilon} \in C^{0}(\bar{\Omega})$.

We also have the following crucial observation. 
Lemma 2.3.

$$
\limsup _{\epsilon \rightarrow 0} \int_{\Omega} V(x) e^{4 \pi(1+\alpha-\epsilon) u_{\epsilon}^{2}} \mathrm{~d} x=\sup _{u \in W_{0}^{1,2}(\Omega),\|u\|_{1, \beta} \leq 1} \int_{\Omega} V(x) e^{4 \pi(1+\alpha) u^{2}} \mathrm{~d} x .
$$

Proof. Obviously,

$$
\limsup _{\epsilon \rightarrow 0} \int_{\Omega} V(x) e^{4 \pi(1+\alpha-\epsilon) u_{\epsilon}^{2}} \mathrm{~d} x \leq \sup _{u \in W_{0}^{1,2}(\Omega),\|u\|_{1, \beta} \leq 1} \int_{\Omega} V(x) e^{4 \pi(1+\alpha) u^{2}} \mathrm{~d} x .
$$

On the other hand, $\forall u \in W_{0}^{1,2}(\Omega)$ with $\|u\|_{1, \beta} \leq 1$, we have by Proposition 2.1,

$$
\int_{\Omega} V(x) e^{4 \pi(1+\alpha) u^{2}} \mathrm{~d} x \leq \liminf _{\epsilon \rightarrow 0} \int_{\Omega} V(x) e^{4 \pi(1+\alpha-\epsilon) u^{2}} \mathrm{~d} x \leq \liminf _{\epsilon \rightarrow 0} \int_{\Omega} V(x) e^{4 \pi(1+\alpha-\epsilon) u_{\epsilon}^{2}} \mathrm{~d} x .
$$

Which implies

$$
\sup _{u \in W_{0}^{1,2}(\Omega),\|u\|_{1, \beta} \leq 1} \int_{\Omega} V(x) e^{4 \pi(1+\alpha) u^{2}} \mathrm{~d} x \leq \liminf _{\epsilon \rightarrow 0} \int_{\Omega} V(x) e^{4 \pi(1+\alpha-\epsilon) u_{\epsilon}^{2}} \mathrm{~d} x .
$$

Hence the result holds.

\section{Blow-up analysis}

In this section, we will develop the blow-up analysis when the sequence $u_{\epsilon}$ blows up when $\epsilon \rightarrow 0$. Since $u_{\epsilon}$ is bounded in $W_{0}^{1,2}(\Omega)$ from the before section, we can assume without loss of the generality

$$
\begin{aligned}
& u_{\epsilon} \rightarrow u_{0} \text { weakly in } W_{0}^{1,2}(\Omega), \\
& u_{\epsilon} \rightarrow u_{0} \text { strongly in } L^{q}(\Omega), \forall q \geq 1, \\
& u_{\epsilon} \rightarrow u_{0} \text { a.e. in } \Omega .
\end{aligned}
$$

Now denote $M_{\epsilon}=\max _{\bar{\Omega}} u_{\epsilon}=u_{\epsilon}\left(x_{\epsilon}\right)$, where $x_{\epsilon} \in \bar{\Omega}$. If $M_{\epsilon}$ is bounded, then for any $u \in W_{0}^{1,2}(\Omega)$ with $\|u\|_{1, \beta} \leq 1$, by the Lebesgue dominated convergence theorem we have

$$
\begin{aligned}
& \int_{\Omega} V(x) e^{4 \pi(1+\alpha) u^{2}} \mathrm{~d} x=\lim _{\epsilon \rightarrow 0} \int_{\Omega} V(x) e^{4 \pi(1+\alpha-\epsilon) u^{2}} \mathrm{~d} x \\
\leq & \lim _{\epsilon \rightarrow 0} \int_{\Omega} V(x) e^{4 \pi(1+\alpha-\epsilon) u_{\epsilon}^{2}} \mathrm{~d} x=\int_{\Omega} V(x) e^{4 \pi(1+\alpha) u_{0}^{2}} \mathrm{~d} x .
\end{aligned}
$$

Hence $u_{0}$ is the desired maximizer.

In the following, we can assume $M_{\epsilon} \rightarrow+\infty$ as $\epsilon \rightarrow 0$. We may also assume $x_{\epsilon} \rightarrow P \in \bar{\Omega}$. Here and in the sequel, we do not distinguish sequence and subsequence, the reader can recognize it from the context. 
In the following, we distinguish two cases (the concentration point $P$ lies in the interior of $\Omega$ or on the boundary of $\Omega$ ) to analyze the asymptotic behavior of $u_{\epsilon}$.

Firstly, by an inequality $e^{t} \leq 1+t e^{t}$,

$$
\int_{\Omega} V(x) \mathrm{d} x<\int_{\Omega} V(x) e^{4 \pi(1+\alpha-\epsilon)} u_{\epsilon}^{2} \mathrm{~d} x \leq \int_{\Omega} V(x) \mathrm{d} x+4 \pi(1+\alpha) \lambda_{\epsilon} .
$$

This leads to $\liminf _{\epsilon \rightarrow 0} \lambda_{\epsilon}>0$.

case 1. $P$ lies in the interior of $\Omega$.

we can prove the concentration-compactness Theorem for $u_{\epsilon}$ near the blow-up point.

Theorem 3.1. $u_{0} \equiv 0$, and $\left|\nabla u_{\epsilon}\right|^{2} \mathrm{~d} x \rightarrow \delta_{P}$, where $\delta_{P}$ denotes the Dirac measure centered at the point $P$.

Proof. Suppose $u_{0} \not \equiv 0$, then we have

$$
\int_{\Omega}\left|\nabla u_{\epsilon}-\nabla u_{0}\right|^{2} \mathrm{~d} x=1-|| u_{0} \|_{1, \beta}^{2}+o_{\epsilon}(1) .
$$

For $p>1, \delta>0, s>1, t>1$ such that $-1<p t \alpha<0$ and $\frac{(1+\alpha)(1+\delta) p s}{1+p \alpha}<1 /\left(1-\left\|u_{0}\right\|_{1, \beta}^{2}\right)$, by using Hölder inequality again, it follow from Lemma 2.1 and Lemma 2.2 to get

$$
\begin{aligned}
& \int_{\Omega} V^{p}(x) u_{\epsilon}^{p} e^{4 \pi(1+\alpha-\epsilon) p u_{\epsilon}^{2}} \mathrm{~d} x \\
\leq & \int_{\Omega} V^{p}(x) u_{\epsilon}^{p} e^{4 \pi(1+\alpha-\epsilon)(1+\delta) p\left(u_{\epsilon}-u_{0}\right)^{2}+4 \pi(1+\alpha-\epsilon) p\left(1+\frac{1}{4 \delta}\right) u_{0}^{2}} \mathrm{~d} x \\
\leq & \left(\int_{\Omega} V^{p}(x) e^{4 \pi(1+\alpha-\epsilon)(1+\delta) p s\left(u_{\epsilon}-u_{0}\right)^{2}}\right)^{\frac{1}{s}} \\
& \times\left(\int_{\Omega} V^{p}(x) u_{\epsilon}^{p s^{*}} e^{4 \pi(1+\alpha-\epsilon) p s^{*}\left(1+\frac{1}{4 \delta}\right) u_{0}^{2}} \mathrm{~d} x\right)^{\frac{1}{s^{*}}} \\
\leq & C\left(\int_{\Omega} V^{p t}(x) e^{4 \pi(1+\alpha-\epsilon) p t s^{*}\left(1+\frac{1}{4 \delta}\right) u_{0}^{2}} \mathrm{~d} x\right)^{\frac{1}{s^{*} t}}\left(\int_{\Omega} u_{\epsilon}^{p s^{*} t^{*}} \mathrm{~d} x\right)^{\frac{1}{s^{*} t^{*}}} \\
< & C,
\end{aligned}
$$

i.e. $\frac{1}{\lambda_{\epsilon}} V(x) u_{\epsilon} e^{4 \pi(1+\alpha-\epsilon) u_{\epsilon}^{2}}$ is uniformly bounded in $L^{p}(\Omega)$ for some $p>1$. By the standard elliptic estimation, we have $u_{\epsilon}$ uniformly bounded in $W_{0}^{2, p}(\Omega)$, and then $u_{\epsilon}$ uniformly bounded in $C(\bar{\Omega})$, which contradicts $M_{\epsilon} \rightarrow+\infty$ as $\epsilon \rightarrow 0$. Hence $u_{0} \equiv 0$.

Since $\int_{\Omega}\left|\nabla u_{\epsilon}\right|^{2} \mathrm{~d} x=1+o_{\epsilon}(1)$ and $u_{\epsilon} \rightarrow 0$ strongly in $L^{q}(\Omega)$ for any $q>1$. Assume $\left|\nabla u_{\epsilon}\right|^{2} \mathrm{~d} x \rightarrow \mu$ in the sense of measure. If $\mu \neq \delta_{P}$, we can choose a cut-off function $\psi(x) \in$ $C_{0}^{1}(\Omega)$, which is supported in $B_{r_{0}}(P) \subset \Omega$ and equal to 1 in $B_{r_{0} / 2}(P)$ for some small $r_{0}>0$ such that

$$
\int_{B_{r_{0}}(P)}\left|\nabla\left(\psi u_{\epsilon}\right)\right|^{2} \mathrm{~d} x \leq 1-\eta
$$


for some $\eta>0$ provided that $\epsilon$ is sufficiently small. Then by Lemma 2.1, Lemma 2.2 and Hölder inequality, $V(x) e^{4 \pi(1+\alpha)\left(\psi u_{\epsilon}\right)^{2}}$ is uniformly bounded in $L^{s}\left(B_{r_{0}}(P)\right)$ for some $s>1$. Then the standard elliptic estimate on the Euler-Lagrange equation (2.3) implies $u_{\epsilon}$ is uniformly bounded in $B_{r_{0} / 2}(P)$, which contradicts $M_{\epsilon} \rightarrow+\infty$. Therefore, $\left|\nabla u_{\epsilon}\right|^{2} \mathrm{~d} x \rightarrow$ $\delta_{P}$.

Next we will locate the position of the point $P$

Subcase 1. $P \in \Omega \backslash\left\{p_{1}, p_{2}, \cdots, p_{m}\right\}$

Let

$$
r_{\epsilon}^{2}=\frac{\lambda_{\epsilon}}{M_{\epsilon}^{2}} e^{-4 \pi(1+\alpha-\epsilon) M_{\epsilon}^{2}}
$$

Since $u_{\epsilon} \rightarrow 0$ in $L^{q}(\Omega)$ for any $q \geq 1$, then we have

$$
r_{\epsilon}^{2} M_{\epsilon}^{2} \rightarrow 0 \quad \text { as } \epsilon \rightarrow 0 .
$$

Choose a ball $B_{\delta}(P)$ such that $p_{i} \notin \overline{B_{\delta}(p)}$ for all $1 \leq i \leq m$.

Denote

$$
B_{0, \epsilon}=\left\{x \in \mathbb{R}^{2}: x_{\epsilon}+r_{\epsilon} x \in B_{\delta}(P)\right\} .
$$

Define the blowing up functions

$$
\psi_{\epsilon}(x)=M_{\epsilon}^{-1} u_{\epsilon}\left(x_{\epsilon}+r_{\epsilon} x\right), \quad \varphi_{\epsilon}(x)=M_{\epsilon}\left(u_{\epsilon}\left(x_{\epsilon}+r_{\epsilon} x\right)-M_{\epsilon}\right) .
$$

A direct computation gives

$$
\begin{aligned}
& -\Delta \psi_{\epsilon}(x)=M_{\epsilon}^{-3} u_{\epsilon}\left(x_{\epsilon}+r_{\epsilon} x\right) V\left(x_{\epsilon}+r_{\epsilon} x\right) e^{4 \pi(1+\alpha-\epsilon)\left(u_{\epsilon}^{2}\left(x_{\epsilon}+r_{\epsilon} x\right)-M_{\epsilon}^{2}\right)}+\beta M_{\epsilon}^{-1} r_{\epsilon}^{2} u_{\epsilon}^{2}\left(x_{\epsilon}+r_{\epsilon} r\right), \\
& -\Delta \varphi_{\epsilon}=M_{\epsilon}^{-1} u_{\epsilon}\left(x_{\epsilon}+r_{\epsilon} x\right) V\left(x_{\epsilon}+r_{\epsilon} x\right) e^{4 \pi(1+\alpha-\epsilon)\left(u_{\epsilon}^{2}\left(x_{\epsilon}+r_{\epsilon} x\right)-M_{\epsilon}^{2}\right)}+\beta M_{\epsilon} r_{\epsilon}^{2} u_{\epsilon}^{2}\left(x_{\epsilon}+r_{\epsilon} r\right),
\end{aligned}
$$

in $B_{0, \epsilon}$. Note that $\left|\psi_{\epsilon}(x)\right| \leq 1$, applying the standard elliptic estimates to above equations, we have $\psi_{\epsilon}(x) \rightarrow \psi_{0}$ in $C_{l o c}^{1}\left(\mathbb{R}^{2}\right)$. In particular

$$
-\Delta \psi_{0}(x)=0 \quad \text { in } \mathbb{R}^{2} .
$$

Liouville-type theorem implies $\psi_{0} \equiv 1$ in $\mathbb{R}^{2}$.

On the other hand, we have in any ball $B_{R}(0)$

$$
u_{\epsilon}^{2}\left(x_{\epsilon}+r_{\epsilon} x\right)-M_{\epsilon}^{2}=2 \varphi_{\epsilon}(x)\left(1+O\left(\psi_{\epsilon}(x)-1\right)\right) .
$$

Applying the standard elliptic estimates to the equation of $\varphi_{\epsilon}$, we also have $\varphi_{\epsilon} \rightarrow \varphi$ in $C_{l o c}^{1}\left(\mathbb{R}^{2}\right)$. In particular,

$$
\left\{\begin{array}{l}
-\Delta \varphi=V(p) e^{8 \pi(1+\alpha) \varphi} \quad \text { in } \mathbb{R}^{2} \\
\varphi(0)=0=\sup \varphi
\end{array}\right.
$$


On one hand, we have for any $R>0$,

$$
\begin{aligned}
& \int_{B_{R}(P)} V(p) e^{8 \pi(1+\alpha) \varphi} \mathrm{d} x=\lim _{\epsilon \rightarrow 0} \int_{B_{R}\left(x_{\epsilon}\right)} V\left(x_{\epsilon}+r_{\epsilon} x\right) e^{4 \pi(1+\alpha-\epsilon)\left(u_{\epsilon}^{2}\left(x_{\epsilon}+r_{\epsilon} x\right)-M_{\epsilon}^{2}\right)} \mathrm{d} x \\
= & \lim _{\epsilon \rightarrow 0} \lambda_{\epsilon}^{-1} \int_{B_{R r_{\epsilon}}\left(x_{\epsilon}\right)} V(y) M_{\epsilon}^{2} e^{4 \pi(1+\alpha-\epsilon) u_{\epsilon}^{2}(y)} \mathrm{d} y \\
= & \lim _{\epsilon \rightarrow 0} \lambda_{\epsilon}^{-1} \int_{B_{R r_{\epsilon}}\left(x_{\epsilon}\right)} V(y) u_{\epsilon}^{2} e^{4 \pi(1+\alpha-\epsilon) u_{\epsilon}^{2}(y)} \mathrm{d} y \leq 1 .
\end{aligned}
$$

This leads to

$$
\int_{\mathbb{R}^{2}} V(P) e^{4 \pi(1+\alpha) \varphi} \mathrm{d} x \leq 1
$$

On the other hand, in view of (3.3) and (3.4), a result of Chen and Li [23] implies that $\varphi$ is radially symmetric

$$
\varphi(x)=-\frac{1}{4 \pi(1+\alpha)} \log \left(1+V(p)(1+\alpha)|x|^{2}\right),
$$

and we get

$$
\int_{\mathbb{R}^{2}} V(P) e^{8 \pi(1+\alpha) \varphi} \mathrm{d} x=\frac{1}{1+\alpha}>1 .
$$

The contradiction between (3.4) and (3.5) indicates that Subcase 1 can not occur. This implies $P \in\left\{p_{1}, p_{2}, \cdots, p_{m}\right\}$.

Subcase 2. $P \in\left\{p_{1}, p_{2}, \cdots, p_{m}\right\}$. Set $P=p_{i}(i=1,2, \cdots, m)$. Let

$$
\rho_{\epsilon}^{2+2 \alpha_{i}}=\frac{\lambda_{\epsilon}}{M_{\epsilon}^{2}} e^{-4 \pi(1+\alpha-\epsilon) M_{\epsilon}^{2}}
$$

Note that $u_{\epsilon} \rightarrow 0$ strongly in $L^{q}(\Omega)$ for any $q>1$. Fix $\tau<1+\alpha$ and choose $s$ close to 1 such that $-\alpha+\tau s<1$, then

$$
\begin{aligned}
& \rho_{\epsilon}^{2+2 \alpha_{i}} e^{4 \pi \tau M_{\epsilon}^{2}} \leq \frac{1}{M_{\epsilon}^{2}} \int_{\Omega} u_{\epsilon}^{2} V(x) e^{4 \pi \tau u_{\epsilon}^{2}} \mathrm{~d} x \\
\leq & \left(\int_{\Omega} u_{\epsilon}^{2 s^{*}} V(x) \mathrm{d} x\right)^{\frac{1}{s^{*}}}\left(\int_{\Omega} V(x) e^{4 \pi \tau s u_{\epsilon}^{2}} \mathrm{~d} x\right)^{\frac{1}{s}} \rightarrow 0 .
\end{aligned}
$$

We obtain

$$
\rho_{\epsilon}^{2+2 \alpha_{i}} e^{4 \pi \tau M_{\epsilon}^{2}} \rightarrow 0, \quad \forall \tau<1+\alpha .
$$

We now distinguish two steps to proceed.

Step 1. $\frac{\left|x_{\epsilon}-p_{i}\right|}{\rho_{\epsilon}} \rightarrow+\infty$ as $\epsilon \rightarrow 0$.

Set $t_{\epsilon}=\rho_{\epsilon}^{1+\alpha_{i}}\left|x_{\epsilon}-p_{i}\right|^{-\alpha_{i}}$ and define $B_{i, \epsilon}=\left\{x \in \mathbb{R}^{2}: x_{\epsilon}+t_{\epsilon} x \in B_{\delta_{i}}\left(p_{i}\right)\right\}$, where $B_{\delta_{i}}\left(p_{i}\right) \subset \Omega$, some $\delta_{i}>0$ and $p_{j} \notin B_{\delta_{i}}\left(p_{i}\right)$ for all $j \neq i$. It is clear that $t_{\epsilon} \rightarrow 0$. We also assume that $V(x)=g_{i}(x)\left|x-p_{i}\right|^{2 \alpha_{i}}$ such that $g_{i}\left(p_{i}\right)>0$. Denote the blowing up functions as

$$
w_{\epsilon}(x)=M_{\epsilon}^{-1} u_{\epsilon}\left(x_{\epsilon}+t_{\epsilon} x\right), \quad v_{\epsilon}(x)=M_{\epsilon}\left(u_{\epsilon}\left(x_{\epsilon}+t_{\epsilon} x\right)-M_{\epsilon}\right) .
$$


A straightforward calculation shows

$$
-\Delta w_{\epsilon}(x)=\frac{u_{\epsilon}(y)}{M_{\epsilon}^{3}} g_{i}(y)\left|x_{\epsilon}-p_{i}\right|^{-2 \alpha_{i}}\left|x_{\epsilon}-p_{i}+t_{\epsilon} x\right|^{2 \alpha_{i}} e^{4 \pi(1+\alpha-\epsilon)\left(u_{\epsilon}^{2}-M_{\epsilon}^{2}\right)}+\beta M_{\epsilon}^{-1} t_{\epsilon}^{2} u_{\epsilon}^{2}(y)
$$

in $B_{i, \epsilon}$, where $y=x_{\epsilon}+t_{\epsilon} x$. Since $0 \leq w_{\epsilon} \leq 1$ and $\left|x_{\epsilon}-p_{i}\right|^{-2 \alpha_{i}}\left|x_{\epsilon}-p_{i}+t_{\epsilon} x\right|^{-\alpha_{i}}-\left.p_{i}\right|^{2 \alpha_{i}}=1+o_{\epsilon}(1)$ and $\beta M_{\epsilon}^{-1} t_{\epsilon}^{2} u_{\epsilon}^{2}(y)=o_{\epsilon}(1)$, where $o_{\epsilon}(1) \rightarrow 0$ in $B_{R}$ for any $R>0$, we have by applying elliptic estimates to (3.6) that $w_{\epsilon} \rightarrow w$ in $C_{l o c}^{1}\left(\mathbb{R}^{2}\right)$, where $w$ satisfies

$$
-\Delta w(x)=0 \quad \text { in } \mathbb{R}^{2}
$$

Since $w \leq 1$ and $w(0)=1$, the Liouville theorem leads to $w=1$. Also we have

$$
-\Delta v_{\epsilon}(x)=\frac{u_{\epsilon}(y)}{M_{\epsilon}} g_{i}(y)\left|x_{\epsilon}-p_{i}\right|^{-2 \alpha_{i}}\left|x_{\epsilon}-p_{i}+t_{\epsilon} x\right|^{2 \alpha_{i}} e^{4 \pi(1+\alpha-\epsilon)\left(u_{\epsilon}^{2}-M_{\epsilon}^{2}\right)}+\beta M_{\epsilon} t_{\epsilon}^{2} u_{\epsilon}^{2}(y) .
$$

Clearly

$$
\frac{u_{\epsilon}(y)}{M_{\epsilon}} g_{i}(y)\left|x_{\epsilon}-p_{i}\right|^{-2 \alpha_{i}}\left|x_{\epsilon}-p_{i}+t_{\epsilon} x\right|^{2 \alpha_{i}} e^{4 \pi(1+\alpha-\epsilon)\left(u_{\epsilon}^{2}-M_{\epsilon}^{2}\right)} \in L_{l o c}^{\infty}\left(B_{i, \epsilon}\right),
$$

and $\beta M_{\epsilon} t_{\epsilon}^{2} u_{\epsilon}^{2}(y)=o_{\epsilon}(1)$. fies

Applying the elliptic estimates to (3.7), we have that $v_{\epsilon} \rightarrow v$ in $C_{l o c}^{1}\left(\mathbb{R}^{2}\right)$, where $v$ satis-

$$
\left\{\begin{array}{l}
-\Delta v=g_{i}\left(p_{i}\right) e^{8 \pi(1+\alpha) v} \quad \text { in } \mathbb{R}^{2}, \\
v(0)=0=\sup _{\mathbb{R}^{2} v} \\
\int_{\mathbb{R}^{2}} g_{i}\left(p_{i}\right) e^{8 \pi(1+\alpha) v} \mathrm{~d} x \leq 1 .
\end{array}\right.
$$

A result of Chen and Li [23] implies that $v$ is radially symmetric and

$$
\int_{\mathbb{R}^{2}} g_{i}\left(p_{i}\right) e^{8 \pi(1+\alpha) v} \mathrm{~d} x=\frac{1}{1+\alpha}>1 .
$$

So we get contradiction, which indicates that the step 1 can not occur.

Step 2. $\frac{\left|x_{\epsilon}-p_{i}\right|}{\rho_{\epsilon}} \leq C$ for some constant $C$. Define

$$
\xi_{\epsilon}(x)=M_{\epsilon}^{-1} u_{\epsilon}\left(x_{\epsilon}+\rho_{\epsilon} x\right), \quad \phi_{\epsilon}(x)=M_{\epsilon}\left(u_{\epsilon}\left(x_{\epsilon}+\rho_{\epsilon} x\right)-M_{\epsilon}\right) .
$$

It follows that $\xi_{\epsilon}(x)$ is a distributional solution to the equation

$$
-\Delta \xi_{\epsilon}(x)=\frac{u_{\epsilon}(y)}{M_{\epsilon}^{3}} g_{i}(y)\left|\frac{x_{\epsilon}-p_{i}}{\rho_{\epsilon}}+x\right|^{2 \alpha_{i}} e^{4 \pi(1+\alpha-\epsilon)\left(u_{\epsilon}^{2}-M_{\epsilon}^{2}\right)}+\beta M_{\epsilon}^{-1} \rho_{\epsilon}^{2} u_{\epsilon}^{2}
$$


in $B_{i, \epsilon}$.

Since $\rho_{\epsilon} \rightarrow 0$ and $B_{2, \epsilon} \rightarrow \mathbb{R}^{2}$, we can assume $\frac{x_{\epsilon}-p_{i}}{\rho_{\epsilon}} \rightarrow x^{*}$ for some $x^{*} \in \mathbb{R}^{2}$. Applying elliptic estimates to (3.8), we have that $\xi_{\epsilon}(x) \rightarrow \xi(x)$ in $C_{l o c}^{1}\left(\mathbb{R}^{2} \backslash\left\{-x^{*}\right\}\right) \cap C_{l o c}^{0}\left(\mathbb{R}^{2}\right)$, where $\xi(x)$ is a distributional harmonic function on $\mathbb{R}^{2}$. Since $\xi(x) \leq \limsup _{\epsilon \rightarrow 0} \xi_{\epsilon}(x) \leq 1$ for all $x \in \mathbb{R}^{2}$ and $\xi(0)=\lim _{\epsilon \rightarrow 0} \xi_{\epsilon}(0)=1$, the Liouville theorem implies that $\xi(x)=1$ on $\mathbb{R}^{2}$. Hence we conclude

$$
\xi_{\epsilon}(x) \rightarrow 1 \quad \text { in } \quad C_{l o c}^{1}\left(\mathbb{R}^{2} \backslash\left\{-x^{*}\right\}\right) \cap C_{l o c}^{0}\left(\mathbb{R}^{2}\right) .
$$

Clearly, $\phi_{\epsilon}$ is a distributional solution to

$$
-\Delta \phi_{\epsilon}(x)=\frac{u_{\epsilon}\left(x_{\epsilon}+\rho_{\epsilon} x\right)}{M_{\epsilon}} g_{i}\left(x_{\epsilon}+\rho_{\epsilon} x\right)\left|x+x^{*}\right|^{2 \alpha_{i}} e^{4 \pi(1+\alpha-\epsilon)\left(u_{\epsilon}^{2}-M_{\epsilon}^{2}\right)}+\beta M_{\epsilon} \rho_{\epsilon}^{2} u_{\epsilon}^{2}
$$

in $B_{i, \epsilon}$. Since $\xi_{\epsilon}(x) \rightarrow 1$ in $C_{l o c}^{1}\left(\mathbb{R}^{2} \backslash\left\{-x^{*}\right\}\right) \cap C_{l o c}^{0}\left(\mathbb{R}^{2}\right)$ and $\phi_{\epsilon}(0)=0=\max _{\mathbb{R}^{2}} \phi_{\epsilon}(x)$, applying elliptic estimates to (3.10), we have that $\phi_{\epsilon}(x) \rightarrow \phi(x)$ in $C_{l o c}^{1}\left(\mathbb{R}^{2} \backslash\left\{-x^{*}\right\}\right) \cap C_{l o c}^{0}\left(\mathbb{R}^{2}\right)$, where $\phi(x)$ is a solution to

$$
-\Delta \phi(x)=g_{i}\left(p_{i}\right)\left|x+x^{*}\right|^{2 \alpha_{i}} e^{8 \pi(1+\alpha) \phi} \quad \text { in } \mathbb{R}^{2} \backslash\left\{-x^{*}\right\} .
$$

If we let $y=x_{\epsilon}+\rho_{\epsilon} x$ with $\left|x+x^{*}\right| \leq R$, then for any fixed $R>\left|x^{*}\right|+1$, there holds $\left|y-p_{i}\right| \leq$ $2 R \rho_{\epsilon}$. Combining (3.9) and Fatou's lemma, we have

$$
\begin{aligned}
& \int_{B_{R}\left(-x^{*}\right)} g_{i}\left(p_{i}\right)\left|x+x^{*}\right|^{2 \alpha_{i}} e^{8 \pi(1+\alpha) \phi} \mathrm{d} x \\
\leq & \limsup _{\epsilon \rightarrow 0} \int_{B_{R}\left(-x^{*}\right)} g_{i}\left(x_{\epsilon}+\rho_{\epsilon} x\right)\left|x+\rho_{\epsilon}^{-1}\left(x_{\epsilon}-p_{i}\right)\right|^{2 \alpha_{i}} e^{4 \pi(1+\alpha-\epsilon)\left(1+\xi_{\epsilon}\right) \phi_{\epsilon}} \mathrm{d} x \\
\leq & \limsup _{\epsilon \rightarrow 0} \lambda_{\epsilon}^{-1} \int_{B_{2 R \rho_{\epsilon}}\left(p_{i}\right)} V(y) u_{\epsilon}^{2} e^{4 \pi(1+\alpha-\epsilon) u_{\epsilon}^{2}(y)} \mathrm{d} y \leq 1 .
\end{aligned}
$$

Hence

$$
\int_{\mathbb{R}^{2}} g_{i}\left(p_{i}\right)\left|x+x^{*}\right|^{2 \alpha_{i}} e^{8 \pi(1+\alpha) \phi} \mathrm{d} x \leq 1 .
$$

By the classification result of J.Prajapat, G.Tarantello [24], we have

$$
\phi(x)=-\frac{1}{4 \pi(1+\alpha)} \log \left(1+g_{i}\left(p_{i}\right) \frac{\pi(1+\alpha)}{\left(1+\alpha_{i}\right)^{2}}\left|x+x^{*}\right|^{2+2 \alpha_{i}}\right) .
$$

Noticing that

$$
\phi(0)=\lim _{\epsilon \rightarrow 0} \phi_{\epsilon}(0)=0,
$$

and combining (3.13) and (3.14), we have that $x^{*}=0$ and thus

$$
\phi(x)=\frac{1}{4 \pi(1+\alpha)} \log \left(1+g_{i}\left(p_{i}\right) \frac{\pi(1+\alpha)}{\left(1+\alpha_{i}\right)^{2}}|x|^{2+2 \alpha_{i}}\right) .
$$


It follows that

$$
\int_{\mathbb{R}^{2}} g_{i}\left(p_{i}\right)|x|^{2 \alpha_{i}} e^{8 \pi(1+\alpha) \phi} \mathrm{d} x=\frac{1+\alpha_{i}}{1+\alpha} \geq 1
$$

The equality holds if and only if $\alpha_{i}=\alpha$. So we have that the blow up point $P$ is the only one point in $\left\{p_{j}\right\}$ with $\alpha_{j}=\alpha$. Without loss of generality, we can assume that the blow up point $P=0$ and $V(x)=g(x)|x|^{2 \alpha}$. Then we have

$$
\int_{\mathbb{R}^{2}} g(0)|x|^{2 \alpha} e^{8 \pi(1+\alpha) \phi(x)} \mathrm{d} x=1 .
$$

Define $u_{\epsilon, L}=\min \left\{u_{\epsilon}, M_{\epsilon} / L\right\}$. Similar to [21], we have the following:

Lemma 3.1. For any $L>1$, there holds

$$
\lim _{\epsilon \rightarrow 0} \int_{\Omega}\left|\nabla u_{\epsilon, L}\right|^{2} \mathrm{~d} x \leq \frac{1}{L}
$$

Proof. In view of the equation (2.3) and by Theorem 3.1, we choose $\left(u_{\epsilon}-\frac{M_{\epsilon}}{L}\right)^{+}$a test function and then obtain

$$
\begin{aligned}
& \int_{\Omega}\left|\nabla\left(u_{\epsilon}-\frac{M_{\epsilon}}{L}\right)^{+}\right|^{2} \mathrm{~d} x=\int_{\Omega} \nabla u_{\epsilon} \nabla\left(u_{\epsilon}-\frac{M_{\epsilon}}{L}\right)^{+} \mathrm{d} x=-\int_{\Omega}\left(u_{\epsilon}-\frac{M_{\epsilon}}{L}\right)^{+} \Delta u_{\epsilon} \mathrm{d} x \\
= & \int_{\Omega}\left(u_{\epsilon}-\frac{M_{\epsilon}}{L}\right)^{+} \frac{u_{\epsilon}(x)}{\lambda_{\epsilon}} V(x) e^{4 \pi(1+\alpha-\epsilon) u_{\epsilon}^{2}(x)} \mathrm{d} x+\beta \int_{\Omega}\left(u_{\epsilon}-\frac{M_{\epsilon}}{L}\right)^{+} u_{\epsilon} \mathrm{d} x \\
\geq & \int_{B_{R \rho_{\epsilon}}\left(x_{\epsilon}\right)}\left(u_{\epsilon}-\frac{M_{\epsilon}}{L}\right)^{+} \frac{u_{\epsilon}(x)}{\lambda_{\epsilon}} V(x) e^{4 \pi(1+\alpha-\epsilon) u_{\epsilon}^{2}(x)} \mathrm{d} x+o_{\epsilon}(1) \\
= & \int_{B_{R}(0)}\left(\frac{u_{\epsilon}}{M_{\epsilon}}-\frac{1}{L}\right)^{+} \frac{u_{\epsilon}\left(x_{\epsilon}+\rho_{\epsilon} y\right)}{M_{\epsilon}}\left|\frac{x_{\epsilon}}{\rho_{\epsilon}}+x\right|^{2 \alpha} g\left(x_{\epsilon}+\rho_{\epsilon} y\right) e^{4 \pi(1+\alpha-\epsilon)\left(u_{\epsilon}^{2}\left(x_{\epsilon}+\rho_{\epsilon} y\right)-M_{\epsilon}^{2}\right)} \mathrm{d} y+o_{\epsilon}(1) \\
\rightarrow & \left(1-\frac{1}{L}\right) \int_{B_{R}(0)} g(0)|x|^{2 \alpha} e^{8 \pi(1+\alpha) \phi(y)} \mathrm{d} y .
\end{aligned}
$$

Taking $R \rightarrow+\infty$, we have form (3.17) that

$$
\liminf _{\epsilon \rightarrow 0} \int_{\Omega}\left|\nabla\left(u_{\epsilon}-\frac{M_{\epsilon}}{L}\right)^{+}\right|^{2} \mathrm{~d} x \geq 1-\frac{1}{L}
$$

Noticing that

$$
\int_{\Omega}\left|\nabla u_{\epsilon, L}\right|^{2} \mathrm{~d} x+\int_{\Omega}\left|\nabla\left(u_{\epsilon}-\frac{M_{\epsilon}}{L}\right)^{+}\right|^{2} \mathrm{~d} x=\left\|u_{\epsilon}\right\|_{1, \beta}+\beta \int_{\Omega} u_{\epsilon}^{2} \mathrm{~d} x=1+o_{\epsilon}(1),
$$

we get the conclusion.

As a consequence of Lemma 3.1, we also have the following: 
Lemma 3.2. There holds

$$
\lim _{\epsilon \rightarrow 0} \int_{\Omega} V(x) e^{4 \pi(1+\alpha-\epsilon) u_{\epsilon}^{2}} \mathrm{~d} x \leq \int_{\Omega} V(x) \mathrm{d} x+\limsup _{\epsilon \rightarrow 0} \frac{\lambda_{\epsilon}}{M_{\epsilon}^{2}} .
$$

Proof. For any $L>1$, there holds

$$
\begin{aligned}
& \int_{\Omega} V(x) e^{4 \pi(1+\alpha-\epsilon) u_{\epsilon}^{2}} \mathrm{~d} x \\
= & \int_{L u_{\epsilon} \leq M_{\epsilon}} V(x) e^{4 \pi(1+\alpha-\epsilon) u_{\epsilon}^{2}} \mathrm{~d} x+\int_{L u_{\epsilon}>M_{\epsilon}} V(x) e^{4 \pi(1+\alpha-\epsilon) u_{\epsilon}^{2}} \mathrm{~d} x \\
\leq & \int_{\Omega} V(x) e^{4 \pi(1+\alpha-\epsilon) u_{\epsilon, L}^{2}} \mathrm{~d} x+\frac{\lambda_{\epsilon} L^{2}}{M_{\epsilon}^{2}} .
\end{aligned}
$$

By Lemmas 3.1 and 2.2, $V(x) e^{4 \pi(1+\alpha-\epsilon) u_{\epsilon, L}^{2}}$ is bounded in $L^{q}(\Omega)$ for some $q>1$. Noticing also that $u_{\epsilon, L}$ convergence to 0 almost everywhere, $V(x) e^{4 \pi(1+\alpha-\epsilon) u_{\epsilon}^{2}}$ converges to $V(x)$ in $L^{1}(\Omega)$. Taking $\epsilon \rightarrow 0$ in (3.18), we obtain

$$
\lim _{\epsilon \rightarrow 0} \int_{\Omega} V(x) e^{4 \pi(1+\alpha-\epsilon) u_{\epsilon}^{2}} \mathrm{~d} x \leq \int_{\Omega} V(x) \mathrm{d} x+L^{2} \limsup _{\epsilon \rightarrow 0} \frac{\lambda_{\epsilon}}{M_{\epsilon}^{2}} .
$$

Let $L \rightarrow 1$, we conclude the Lemma.

It follows Lemma 3.2 that

$$
\limsup _{\epsilon \rightarrow 0} \frac{\lambda_{\epsilon}}{M_{\epsilon}^{\theta}}=+\infty, \quad \forall \theta<2
$$

For otherwise, we have $\lambda_{\epsilon} / M_{\epsilon}^{2} \rightarrow 0$ as $\epsilon \rightarrow 0$. Let $v \in W_{0}^{1,2}(\Omega)$ be such that $\|v\|_{1, \beta}=1$. Then we have by Lemma 3.2 that

$$
\begin{aligned}
& \int_{\Omega} V(x) e^{4 \pi(1+\alpha) v^{2}} \mathrm{~d} x \leq \sup _{u \in W_{0}^{1,2}(\Omega),\|\nabla u\|_{L^{2}(\Omega)} \leq 1} \int_{\Omega} V(x) e^{4 \pi(1+\alpha) u^{2}} \mathrm{~d} x \\
= & \lim _{\epsilon \rightarrow 0} \int_{\Omega} V(x) e^{4 \pi(1+\alpha-\epsilon) u_{\epsilon}^{2}} \mathrm{~d} x=\int_{\Omega} V(x) \mathrm{d} x .
\end{aligned}
$$

This is impossible since $v \not \equiv 0$. Thus (3.19) holds.

The following Lemma will be used in Section 5 .

\section{Lemma 3.3.}

$$
\lim _{\epsilon \rightarrow 0} \int_{\Omega} V(x) e^{4 \pi(1+\alpha-\epsilon) u_{\epsilon}^{2}} \mathrm{~d} x=\int_{\Omega} V(x) \mathrm{d} x+\lim _{R \rightarrow+\infty} \limsup _{\epsilon \rightarrow 0} \int_{B_{R \rho_{\epsilon}}\left(x_{\epsilon}\right)} V(x) e^{4 \pi(1+\alpha-\epsilon) u_{\epsilon}^{2}} \mathrm{~d} x .
$$


Proof. On the one hand,

$$
\begin{aligned}
& \limsup _{\epsilon \rightarrow 0} \int_{B_{R \rho_{\epsilon}}\left(x_{\epsilon}\right)} V(x) e^{4 \pi(1+\alpha-\epsilon) u_{\epsilon}^{2}} \mathrm{~d} x \\
\leq & \limsup _{\epsilon \rightarrow 0} \int_{\Omega} V(x) e^{4 \pi(1+\alpha-\epsilon) u_{\epsilon}^{2}} \mathrm{~d} x-\liminf _{\epsilon \rightarrow 0} \int_{\Omega \backslash B_{R \rho_{\epsilon}}\left(x_{\epsilon}\right)} V(x) e^{4 \pi(1+\alpha-\epsilon) u_{\epsilon}^{2}} \mathrm{~d} x \\
\leq & \limsup _{\epsilon \rightarrow 0} \int_{\Omega} V(x) e^{4 \pi(1+\alpha-\epsilon) u_{\epsilon}^{2}} \mathrm{~d} x-\int_{\Omega} V(x) \mathrm{d} x .
\end{aligned}
$$

On the other hand,

$$
\int_{B_{R \rho_{\epsilon}}\left(x_{\epsilon}\right)} V(x) e^{4 \pi(1+\alpha-\epsilon) u_{\epsilon}^{2}} \mathrm{~d} x=\frac{\lambda_{\epsilon}}{M_{\epsilon}^{2}}\left(\int_{B_{R}(0)} g(0)|x|^{2 \alpha} e^{8 \pi(1+\alpha) \phi(x)} \mathrm{d} x+o_{\epsilon}(1)\right),
$$

which gives

$$
\lim _{R \rightarrow+\infty} \limsup _{\epsilon \rightarrow 0} \int_{B_{R \rho_{\epsilon}}\left(x_{\epsilon}\right)} V(x) e^{4 \pi(1+\alpha-\epsilon) u_{\epsilon}^{2}} \mathrm{~d} x=\limsup _{\epsilon \rightarrow 0} \frac{\lambda_{\epsilon}}{M_{\epsilon}^{2}} .
$$

Combining (3.20), (3.21), Lemma 3.2 and Lemma 2.3, we get the result.

In order to investigate the convergence behavior of $u_{\epsilon}$ away from $P=0$, we need the following Lemma.

Lemma 3.4. We have

$$
\lim _{\epsilon \rightarrow 0} \int_{\Omega} \frac{M_{\epsilon} u_{\epsilon}}{\lambda_{\epsilon}} V(x) e^{4 \pi(1+\alpha-\epsilon) u_{\epsilon}^{2}} \mathrm{~d} x=1 .
$$

Proof. We divide $\Omega$ into three parts

$$
\Omega=\left(\left\{u_{\epsilon}>\frac{M_{\epsilon}}{L}\right\} \backslash B_{R \rho_{\epsilon}}\left(x_{\epsilon}\right)\right) \cup\left\{u_{\epsilon} \leq \frac{M_{\epsilon}}{L}\right\} \cup B_{R \rho_{\epsilon}}\left(x_{\epsilon}\right)
$$

for some $L>1$ and any $R>0$. Denote the integral on the above domains by $I_{1}, I_{2}$ and $I_{3}$ respectively. Since

$$
\begin{aligned}
I_{1} & \leq L \int_{\left\{u_{\epsilon}>\frac{M_{\epsilon}}{L}\right\} \backslash B_{R \rho_{\epsilon}}\left(x_{\epsilon}\right)} \frac{u_{\epsilon}^{2}}{\lambda_{\epsilon}} V(x) e^{4 \pi(1+\alpha-\epsilon) u_{\epsilon}^{2}} \mathrm{~d} x \\
& \leq L \int_{\Omega \backslash B_{R \rho_{\epsilon}}\left(x_{\epsilon}\right)} \frac{u_{\epsilon}^{2}}{\lambda_{\epsilon}} V(x) e^{4 \pi(1+\alpha-\epsilon) u_{\epsilon}^{2}} \mathrm{~d} x \\
& =L-L \int_{B_{R \rho_{\epsilon}}\left(x_{\epsilon}\right)} \frac{u_{\epsilon}^{2}}{\lambda_{\epsilon}} V(x) e^{4 \pi(1+\alpha-\epsilon) u_{\epsilon}^{2}} \mathrm{~d} x \\
& \rightarrow L\left(1-\int_{B_{R}(0)} g(0)|x|^{2 \alpha} e^{8 \pi(1+\alpha-\epsilon) \phi(x)} \mathrm{d} x\right),
\end{aligned}
$$

then we have

$$
\lim _{R \rightarrow+\infty \epsilon \rightarrow 0} \lim _{\epsilon} I_{1}=0 .
$$


For $I_{2}$, it is easy to get

$$
I_{2}=\frac{M_{\epsilon}}{\lambda_{\epsilon}} \int_{\Omega} u_{\epsilon, L} e^{4 \pi(1+\alpha-\epsilon) u_{\epsilon, L}^{2}} \mathrm{~d} x \rightarrow 0
$$

For $I_{3}$, since

$$
\begin{aligned}
I_{3} & =\int_{B_{R}} \frac{u_{\epsilon}\left(x_{\epsilon}+\rho_{\epsilon} x\right)}{M_{\epsilon}} g\left(x_{\epsilon}+\rho_{\epsilon} x\right)\left|\frac{x_{\epsilon}}{\rho_{\epsilon}}+x\right|^{2 \alpha} e^{4 \pi(1+\alpha-\epsilon)\left(u_{\epsilon}^{2}\left(x_{\epsilon}+\rho_{\epsilon} x\right)-M_{\epsilon}^{2}\right)} \mathrm{d} x \\
& \rightarrow \int_{B_{R}} g(0)|x|^{2 \alpha} e^{8 \pi(1+\alpha) \phi(x)} \mathrm{d} x,
\end{aligned}
$$

then we have

$$
\lim _{R \rightarrow+\infty \epsilon \rightarrow 0} \lim _{\epsilon} I_{3}=1 .
$$

Putting (3.23)-(3.25) together, the result holds.

Let $g_{\epsilon}=M_{\epsilon} u_{\epsilon}$. It is clear that $g_{\epsilon}$ satisfies the following equation

$$
-\Delta g_{\epsilon}+\beta g_{\epsilon}=\frac{g_{\epsilon}}{\lambda_{\epsilon}} V(x) e^{4 \pi(1+\alpha-\epsilon) u_{\epsilon}^{2}} \quad \text { in } \mathcal{D}^{\prime}(\Omega) .
$$

Eq. (3.22) and its proof show that $\frac{g_{\epsilon}}{\lambda_{\epsilon}} V(x) e^{4 \pi(1+\alpha-\epsilon) u_{\epsilon}^{2}}$ converges to the Dirac operator $\delta_{0}$ in $\mathcal{D}^{\prime}(\Omega)$. This suggests that $g_{\epsilon}$ should tend to the corresponding Green's function $G$. That is confirmed as follows.

Lemma 3.5. $g_{\epsilon}$ is uniformly bounded in $W_{0}^{1, q}(\Omega)$ for any $1<q<2$. Furthermore, $g_{\epsilon} \rightarrow G$ weakly in $W_{0}^{1, q}(\Omega)$ for any $1<q<2$ and $g_{\epsilon} \rightarrow G$ uniformly in $C_{l o c}^{0}(\bar{\Omega} \backslash\{0\})$, where G satisfies

$$
\begin{cases}-\Delta G+\beta G=\delta_{0} & x \in \Omega, \\ G=0 & x \in \partial \Omega,\end{cases}
$$

in the distributional sense.

Proof. It follows from (3.22) that $\frac{M_{\epsilon} u_{\epsilon}}{\lambda_{\epsilon}} V(x) e^{4 \pi(1+\alpha-\epsilon) u_{\epsilon}^{2}}$ is uniformly bounded in $L^{1}(\Omega)$. We claim that $g_{\epsilon}$ is uniformly bounded in $L^{1}(\Omega)$. To see this, we suppose on the contrary that $\left\|g_{\epsilon}\right\|_{L^{1}(\Omega)} \rightarrow+\infty$ as $\epsilon \rightarrow 0$. Set $f_{\epsilon}=\frac{g_{\epsilon}}{\left\|g_{\epsilon}\right\|_{L^{1}(\Omega)}}$, and we have $\left\|f_{\epsilon}\right\|_{L^{1}(\Omega)}=1$. Then applying a result of Struwe ([26, Theorem 2.2]), we have that $f_{\epsilon}$ is uniformly bounded in $W_{0}^{1, q}(\Omega)$ for any $1<q<2$, in particular $f_{\epsilon} \rightarrow f$ strongly in $L^{1}(\Omega)$. Since

$$
\frac{1}{\left\|g_{\epsilon}\right\|_{L^{1}(\Omega)}} \frac{M_{\epsilon} u_{\epsilon}}{\lambda_{\epsilon}} V(x) e^{4 \pi(1+\alpha-\epsilon) u_{\epsilon}^{2}} \rightarrow 0
$$

in $L^{1}(\Omega), f$ is a distributional solution to $-\Delta f+\beta f=0$ in $\Omega$, which leads to $f \equiv 0$. This contradicts

$$
\|f\|_{L^{1}(\Omega)}=\lim _{\epsilon \rightarrow 0}|| f_{\epsilon} \|_{L^{1}(\Omega)}=1
$$


and confirms our claim. Now since $\frac{M_{\epsilon} u_{\epsilon}}{\lambda_{\epsilon}} V(x) e^{4 \pi(1+\alpha-\epsilon) u_{\epsilon}^{2}}-\beta g_{\epsilon}$ is uniformly bounded in $L^{1}(\Omega)$, applying Struwe ([26, Theorem 2.2]) again, we have that $g_{\epsilon}$ is uniformly bounded in $W_{0}^{1, q}(\Omega)$ for any $1<q<2$. Hence there exist $G \in \cap_{1<q<2} W_{0}^{1, q}(\Omega)$ such that $g_{\epsilon} \rightarrow G$ weakly in $W_{0}^{1, q}(\Omega)$ for any $1<q<2$. Since (3.22) implies that $\frac{M_{\varepsilon} u_{\epsilon}}{\lambda_{\epsilon}} V(x) e^{4 \pi(1+\alpha-\epsilon) u_{\epsilon}^{2}} \rightarrow \delta_{0}$ in sense of measure. Then $G$ is a distributional solution to (3.27). Sobolev embeding implies $g_{\epsilon} \rightarrow G$ in $L^{t}(\Omega)$ for any $t>1$.

Moreover, using the Hölder inequality and Lemma 2.1, for $p>1, r>1, q>1$ such that $-1<p q \alpha<0$ and $\operatorname{pr}(1+\alpha) \leq 1+\alpha p$, we can have that

$$
\begin{aligned}
& \int_{\Omega}(V(x))^{p}\left(g_{\epsilon}\right)^{p} e^{4 \pi p(1+\alpha-\epsilon) u_{\epsilon}^{2}} \mathrm{~d} x \\
\leq & \left(\int_{\Omega}(V(x))^{p}\left(g_{\epsilon}\right)^{r^{*} p} \mathrm{~d} x\right)^{\frac{1}{r^{*}}}\left(\int_{\Omega}(V(x))^{p} e^{4 \pi p r(1+\alpha-\epsilon) u_{\epsilon}^{2}} \mathrm{~d} x\right)^{\frac{1}{r}} \\
\leq & \left(\int_{\Omega}(V(x))^{p q} \mathrm{~d} x\right)^{\frac{1}{q r^{*}}}\left(\int_{\Omega} g_{\epsilon}^{r^{*} p q^{*}} \mathrm{~d} x\right)^{\frac{1}{r^{*} q^{*}}}\left(\int_{\Omega}(V(x))^{p} e^{4 \pi p r(1+\alpha-\epsilon) u_{\epsilon}^{2}} \mathrm{~d} x\right)^{\frac{1}{r}} \\
< & +\infty .
\end{aligned}
$$

So $\frac{1}{\lambda_{\epsilon}} V(x) g_{\epsilon} e^{4 \pi(1+\alpha-\epsilon)} u_{\epsilon}^{2}$ is uniformly bounded in $L^{p}(\Omega)$ for some $p>1$. By using the standard elliptic estimation to the (3.26), then $g_{\epsilon}$ is uniformly bounded in $W_{0}^{2, p}(\Omega)$. The Sobolev embeding Theorem implies $g_{\epsilon} \rightarrow G$ uniformly in $C_{l o c}^{0}(\bar{\Omega} \backslash\{0\})$.

Obviously, $G$ takes the form

$$
G(x)=-\frac{1}{2 \pi} \log |x|+C_{G}+\psi(x),
$$

where $C_{G}$ is a constant and $\psi \in C^{1}(\bar{\Omega})$.

Up to now, we have described the convergence behavior of $u_{\epsilon}$ near $P$ and away from $P$ when the concentration point $P$ is in the interior of $\Omega$.

Case 2. $P$ lies on $\partial \Omega$.

Lemma 3.6. Let $d_{\epsilon}=\operatorname{dist}\left(x_{\epsilon}, \partial \Omega\right)$, and $r_{\epsilon}$ be defined in (3.2). There holds $r_{\epsilon} / d_{\epsilon} \rightarrow 0$.

Proof. We suppose on the contrary that there exists $R>0$ such that $d_{\epsilon} \leq R r_{\epsilon}$. Take some $y_{\epsilon} \in \partial \Omega$ such that $d_{\epsilon}=\left|x_{\epsilon}-y_{\epsilon}\right|$. Set $B_{\delta}=B_{\delta}(P) \cap \Omega$ with some $\delta>0$ such that $p_{i} \notin \overline{B_{\delta}}$ for all $1 \leq i \leq m$ and

$$
B_{\delta, \epsilon}=\left\{x \in \mathbb{R}^{2} \mid x_{\epsilon}+r_{\epsilon} x \in B_{\delta}\right\} .
$$

Then $B_{\delta, \epsilon} \rightarrow \mathbb{R}_{+}^{2}\left(t_{0}\right):=\left\{\left(x_{1}, x_{2}\right) \in \mathbb{R}^{2}: x_{1}>t_{0}\right\}$ as $\epsilon \rightarrow 0$. Let $\bar{v}_{\epsilon}=M_{\epsilon}^{-1} u_{\epsilon}\left(y_{\epsilon}+r_{\epsilon} x\right)$. In this case $\bar{v}_{\epsilon} \rightarrow \bar{v}$ in $C_{l o c}^{1}\left(\overline{\mathbb{R}}_{+}^{2}\left(t_{0}\right)\right)$ and $\bar{v}$ satisfies

$$
\begin{cases}-\Delta \bar{v}=0 & x \in \mathbb{R}_{+}^{2}\left(t_{0}\right), \\ \bar{v}=0 & x \in \partial \mathbb{R}_{+}^{2}\left(t_{0}\right) .\end{cases}
$$


By a reflection argument, we have $-\Delta \bar{v}=0$ in $\mathbb{R}^{2}$. For $\left\|\bar{v}_{\epsilon}\right\|_{L^{\infty}\left(\overline{B_{\delta, \epsilon}}\right)}=1$, we can easily get that $\bar{v}=1$. Then we have $\bar{v}_{\epsilon} \rightarrow 1$ in $C_{l o c}^{1}\left(\mathbb{R}_{+}^{2}\left(t_{0}\right)\right)$. This contradicts to the fact $\bar{v}_{\epsilon}(0)=0$.

By Lemma 3.6, we have $\lim _{\epsilon \rightarrow 0} \frac{\operatorname{dist}\left(x_{\epsilon}, \partial \Omega\right)}{r_{\epsilon}} \rightarrow+\infty$, and $B_{\delta, \epsilon} \rightarrow \mathbb{R}^{2}$ as $\epsilon \rightarrow 0$. Define the blowing up functions

$$
\begin{array}{ll}
\bar{\psi}_{\epsilon}=M_{\epsilon}^{-1} u_{\epsilon}\left(x_{\epsilon}+r_{\epsilon} x\right) & \text { in } B_{\delta, \epsilon} \\
\bar{\varphi}_{\epsilon}=M_{\epsilon}\left(u_{\epsilon}\left(x_{\epsilon}+r_{\epsilon} x\right)-M_{\epsilon}\right) & \text { in } B_{\delta, \epsilon} .
\end{array}
$$

Similar to Subcase $1, \bar{\psi}_{\epsilon} \rightarrow 1$ in $C_{l o c}^{1}\left(\mathbb{R}^{2}\right)$, and $\bar{\varphi}_{\epsilon} \rightarrow \bar{\varphi}$ in $C_{l o c}^{1}\left(\mathbb{R}^{2}\right) . \bar{\varphi}$ satisfy

$$
\left\{\begin{array}{l}
-\Delta \bar{\varphi}(x)=V(P) e^{8 \pi(1+\alpha) \bar{\varphi}} \quad \text { in } \quad \mathbb{R}^{2} \\
\bar{\varphi}(0)=0=\sup _{\mathbb{R}^{2}} \bar{\varphi} \\
\int_{\mathbb{R}^{2}} V(0) e^{8 \pi(1+\alpha) \bar{\varphi}} \mathrm{d} x \leq 1
\end{array}\right.
$$

By the classify of solution, we have

$$
\bar{\varphi}(x)=-\frac{1}{4 \pi(1+\alpha)} \log \left(1+V(p)(1+\alpha)|x|^{2}\right),
$$

and we get

$$
\int_{\mathbb{R}^{2}} V(P) e^{8 \pi(1+\alpha) \bar{\varphi}} \mathrm{d} x=\frac{1}{1+\alpha}>1 .
$$

Thus we get a contradiction. So the case don't occur.

\section{Proof of theorem}

In this section,we show extremal functions exist. To this purpose, we need to derive the upper bound first. We also need the following similar result motivated by the arguments by Carleson and Chang in [6].

Definition 4.1. For $n \geq 2$. A sequence $\left\{u_{\epsilon}\right\} \subset W_{0}^{1,2}(\Omega)$ is a normalized concentrating sequence if

(i) $\int_{\Omega}\left|\nabla u_{\epsilon}\right|^{2} \mathrm{~d} x=1$,

(ii) $u_{\epsilon} \rightarrow 0$ weakly in $W_{0}^{1,2}(\Omega)$,

(iii) there exist $x_{0} \in \Omega$ such that any $r>0, \int_{\Omega \backslash B_{r}\left(x_{0}\right)}\left|\nabla u_{\epsilon}\right|^{2} \mathrm{~d} x \rightarrow 0$ as $\epsilon \rightarrow 0$.

Then we call $u_{\epsilon}$ as a normalized concentrating sequences, and $x_{0}$ is called as a blow up point. 
Theorem 4.1. Assume that $\left\{u_{\epsilon}\right\} \subset W_{0}^{1,2}\left(B_{\rho}(0)\right)$ is a normalized concentrating sequence with a blow up point, the origin. Then for $\alpha \in(-1,0]$, we have

$$
\limsup _{\epsilon \rightarrow 0} \int_{B_{\rho}(0)}|x|^{n \alpha}\left(e^{4 \pi(1+\alpha) u_{\epsilon}^{2}}-1\right) \mathrm{d} x \leq \frac{\pi \rho^{2+2 \alpha}}{1+\alpha} e .
$$

Proof. Let $u_{\epsilon}^{*}$ is the schwarz symmetry rearrangement of the function $u_{\epsilon}(x)$. Set $\psi_{\epsilon}(x)=$ $(1+\alpha)^{\frac{1}{2}} u_{\epsilon}^{*}\left(|x|^{\frac{1}{1+\alpha}}\right)$, then we have

$$
\int_{B_{\rho}(0)}\left|\nabla \psi_{\epsilon}\right|^{2} \mathrm{~d} x=\int_{B_{\rho}(0)}\left|\nabla u_{\epsilon}^{*}\right|^{2} \mathrm{~d} x \leq \int_{B_{\rho}(0)}\left|\nabla u_{\epsilon}\right|^{2} \mathrm{~d} x=1 .
$$

Moreover, by Hardy-Littlewood inequality,

$$
\begin{aligned}
& \int_{B_{\rho}(0)}|x|^{n \alpha}\left(e^{4 \pi(1+\alpha)\left|u_{\epsilon}(x)\right|^{2}}-1\right) \mathrm{d} x \leq \int_{B_{\rho}(0)}|x|^{2 \alpha}\left(e^{4 \pi(1+\alpha)\left|u_{\epsilon}^{*}(x)\right|^{2}}-1\right) \mathrm{d} x \\
= & \frac{\rho^{2 \alpha}}{1+\alpha} \int_{B_{\rho}(0)}\left(e^{4 \pi \psi_{\epsilon}^{2}}-1\right) \mathrm{d} x .
\end{aligned}
$$

Now we use a fact in [6]: For any normalized concentrating sequence $\left\{\psi_{\epsilon}\right\} \in W_{0}^{1,2}\left(B_{\rho}(0)\right)$ it holds

$$
\limsup _{\epsilon \rightarrow 0} \int_{B_{\rho}(0)}\left(e^{4 \pi \psi_{\epsilon}^{2}}-1\right) \mathrm{d} x \leq \pi \rho^{2} e .
$$

Hence (4.1) holds.

Set

$$
T_{0}=\sup _{u \in W_{0}^{1,2}(\Omega),\|u\|_{1, \beta} \leq 1} \int_{\Omega} V(x) e^{4 \pi(1+\alpha) u^{2}} \mathrm{~d} x .
$$

Motivated by the arguments in [6] and [27], we first compute the upper bound of $T_{0}$ if $u_{\epsilon}$ blows up. Our Lemma is the following:

Lemma 4.1. If $\limsup _{\epsilon \rightarrow 0}\left\|u_{\epsilon}\right\|_{\infty}=\infty$, then

$$
T_{0} \leq \int_{\Omega} V(x) \mathrm{d} x+\frac{g(0) \pi}{1+\alpha} e^{4 \pi(1+\alpha) C_{G}+1} .
$$

Proof.

$$
\begin{aligned}
\int_{\Omega \backslash B_{\delta}(0)}|\nabla G|^{2} \mathrm{~d} x & =-\int_{\partial B_{\delta}(0)} G \frac{\partial G}{\partial v} \mathrm{~d} s+\int_{\partial \Omega} G \frac{\partial G}{\partial v} \mathrm{~d} s-\int_{\Omega \backslash B_{\delta}(0)} \Delta G \mathrm{~d} x \\
& =-\int_{\partial B_{\delta}(0)} G \frac{\partial G}{\partial v} \mathrm{~d} s+\beta \int_{\Omega \backslash B_{\delta}(0)} G^{2} \mathrm{~d} x \\
& =-\frac{1}{2 \pi} \log \delta+C_{G}+\beta\|G\|_{L^{2}(\Omega)}^{2}+o_{\delta}(1),
\end{aligned}
$$


where $o_{\delta}(1) \rightarrow 0$ as $\delta \rightarrow 0$. Hence we obtain

$$
\int_{\Omega \backslash B_{\delta}(0)}\left|\nabla u_{\epsilon}\right|^{2} \mathrm{~d} x=\frac{1}{M_{\epsilon}^{2}}\left(-\frac{1}{2 \pi} \log \delta+C_{G}+\beta\|G\|_{L^{2}(\Omega)}^{2}+o_{\delta}(1)+o_{\epsilon}(1)\right),
$$

where $o_{\epsilon}(1) \rightarrow 0$ as $\epsilon \rightarrow 0$. Let $b_{\epsilon}=\sup _{\partial B_{\delta}(0)} u_{\epsilon}$ and $\bar{u}_{\epsilon}=\left(u_{\epsilon}-b_{\epsilon}\right)^{+}$. Then $\bar{u}_{\epsilon} \in W_{0}^{1,2}\left(B_{\delta}(0)\right)$, by (4.3) and the fact that

$$
\int_{B_{\delta}(0)}\left|\nabla u_{\epsilon}\right|^{2} \mathrm{~d} x=1-\int_{\Omega \backslash B_{\delta}(0)}\left|\nabla u_{\epsilon}\right|^{2} \mathrm{~d} x+\beta \int_{\Omega} u_{\epsilon}^{2} \mathrm{~d} x
$$

we have

$$
\int_{B_{\delta}(0)}\left|\nabla \bar{u}_{\epsilon}\right|^{2} \mathrm{~d} x:=\tau_{\epsilon} \leq 1-\frac{1}{M_{\epsilon}^{2}}\left(-\frac{1}{2 \pi} \log \delta+C_{G}+o_{\delta}(1)+o_{\epsilon}(1)\right) .
$$

By Theorem 4.1,

$$
\limsup _{\epsilon \rightarrow 0} \int_{B_{\delta}(0)}|x|^{2 \alpha}\left(e^{4 \pi(1+\alpha) \frac{\bar{u}_{\epsilon}^{2}}{\tau_{\epsilon}}}-1\right) \mathrm{d} x \leq \frac{\pi \delta^{2+2 \alpha}}{1+\alpha} e .
$$

Then if we set $g(\xi)=\max _{x \in \bar{B}_{\delta}(0)}\{g(x)\}$ with $\xi \in \bar{B}_{\delta}(0)$, we have

$$
\limsup _{\epsilon \rightarrow 0} \int_{B_{\delta}(0)} g(x)|x|^{2 \alpha}\left(e^{4 \pi(1+\alpha) \frac{\bar{\eta}_{\epsilon}^{2}}{\tau_{\epsilon}}}-1\right) \mathrm{d} x \leq \frac{g(\xi) \pi \delta^{2+2 \alpha}}{1+\alpha} e
$$

Now we focus the estimate on bubbling domain $B_{R \rho_{\epsilon}}\left(x_{\epsilon}\right)$. By Step 2, $\phi_{\epsilon} \rightarrow \phi$ in $C_{l o c}^{1}\left(\mathbb{R}^{n}\right)$, and whence $u_{\epsilon}=M_{\epsilon}+o_{\epsilon}(R)$, where $o_{\epsilon}(R) \rightarrow 0$ as $\epsilon \rightarrow 0$ for any fixed $R>0$. Together with the fact $M_{\epsilon} u_{\epsilon} \rightarrow G$ strongly in $C_{l o c}^{0}(\bar{\Omega} \backslash\{0\})$, we have

$$
4 \pi(1+\alpha-\epsilon) u_{\epsilon}^{2} \leq 4 \pi(1+\alpha)\left(\bar{u}_{\epsilon}+b_{\epsilon}\right)^{2}=4 \pi(1+\alpha) \bar{u}_{\epsilon}^{2}+8 \pi(1+\alpha) \bar{u}_{\epsilon} b_{\epsilon}+4 \pi(1+\alpha) b_{\epsilon}^{2},
$$

and

$$
\bar{u}_{\epsilon} b_{\epsilon}=-\frac{1}{2 \pi} \log \delta+C_{G}+o_{\delta}(1)+o_{\epsilon}(1) .
$$

Notice that

$$
\begin{aligned}
4 \pi(1+\alpha) \bar{u}_{\epsilon}^{2} & \leq \frac{4 \pi(1+\alpha) \bar{u}_{\epsilon}^{2}}{\tau_{\epsilon}}-4 \pi(1+\alpha)\left(-\frac{1}{2 \pi} \log \delta+C_{G}+o_{\delta}(1)+o_{\epsilon}(1)\right) \\
& =\frac{4 \pi(1+\alpha) \bar{u}_{\epsilon}^{2}}{\tau_{\epsilon}}+2(1+\alpha) \log \delta-4 \pi(1+\alpha) C_{G}+o_{\delta}(1)+o_{\epsilon}(1) .
\end{aligned}
$$

Putting (4.5)-(4.7) together, we obtain on $B_{R \rho_{\epsilon}}\left(x_{\epsilon}\right)$

$$
4 \pi(1+\alpha-\epsilon) u_{\epsilon}^{2} \leq \frac{4 \pi(1+\alpha) \bar{u}_{\epsilon}^{2}}{\tau_{\epsilon}}-2(1+\alpha) \log \delta+4 \pi(1+\alpha) C_{G}+o_{\delta}(1)+o_{\epsilon}(1) .
$$


Therefore, we have

$$
\begin{aligned}
& \quad \limsup _{\epsilon \rightarrow 0} \int_{B_{R \rho \epsilon}(x)} V(x) e^{4 \pi(1+\alpha-\epsilon) u_{\epsilon}^{2}} \mathrm{~d} x \\
& \leq \delta^{-2(1+\alpha)} e^{4 \pi(1+\alpha) C_{G}+o_{\delta}(1)} \limsup _{\epsilon \rightarrow 0} \int_{B_{R \rho_{\epsilon}\left(x_{\epsilon}\right)}} g(x)|x|^{2 \alpha} e^{4 \pi(1+\alpha) \frac{\bar{u}_{\epsilon}^{2}}{\tau_{\epsilon}}} \mathrm{d} x \\
& \leq \delta^{-2(1+\alpha)} e^{4 \pi(1+\alpha) C_{G}+o_{\delta}(1)} \limsup _{\epsilon \rightarrow 0} \int_{B_{R \rho_{\epsilon}}\left(x_{\epsilon}\right)} g(x)|x|^{2 \alpha}\left(e^{4 \pi(1+\alpha) \frac{\bar{\epsilon}_{\epsilon}^{2}}{\tau_{\epsilon}}}-1\right) \mathrm{d} x \\
& \leq \delta^{-2(1+\alpha)} e^{4 \pi(1+\alpha) C_{G}+o_{\delta}(1)} \limsup _{\epsilon \rightarrow 0} \int_{B_{\delta}(0)} g(x)|x|^{2 \alpha}\left(e^{4 \pi(1+\alpha) \frac{\bar{u}_{\epsilon}^{2}}{\tau_{\epsilon}}}-1\right) \mathrm{d} x \\
& \leq \delta^{-2(1+\alpha)} e^{4 \pi(1+\alpha) C_{G}+o_{\delta}(1)} \frac{g(\xi) \pi \delta^{2+2 \alpha}}{1+\alpha} e .
\end{aligned}
$$

Taking $\delta \rightarrow 0$, by the continuity of $g(x)$ near the origin, we have

$$
\limsup _{\epsilon \rightarrow 0} \int_{B_{R \rho_{\epsilon}\left(x_{\epsilon}\right)}} V(x) e^{4 \pi(1+\alpha-\epsilon) u_{\epsilon}^{2}} \mathrm{~d} x \leq \frac{g(0) \pi}{1+\alpha} e^{4 \pi(1+\alpha) C_{G}+1} .
$$

Then by the Lemma 3.3, we have

$$
\limsup _{\epsilon \rightarrow 0} \int_{\Omega} V(x) e^{4 \pi(1+\alpha-\epsilon) u_{\epsilon}^{2}} \leq \int_{\Omega} V(x) \mathrm{d} x+\frac{g(0) \pi}{1+\alpha} e^{4 \pi(1+\alpha) C_{G}+1} .
$$

The proof is completed by using Lemma 2.3.

The proof of Theorem 1.1 If the sequence $\left\|u_{\epsilon}\right\|_{L^{\infty}(\Omega)}$ is uniformly bounded, by Lemma 2.3, we can easily get $T_{0}<+\infty$. While if $\left\|u_{\epsilon}\right\|_{L^{\infty}(\Omega)} \rightarrow+\infty$, then by Lemma 4.1 we also get $T_{0}<+\infty$.

Next we construct a sequence to get the lower bound estimate, which is an opposite to $T_{0}$. Thus we get a contradiction and consequently we complete the proof of Theorem 1.2 .

Lemma 4.2. There holds

$$
T_{0}>\int_{\Omega} V(x) \mathrm{d} x+\frac{g(0) \pi}{1+\alpha} e^{4 \pi(1+\alpha) C_{G}+1} .
$$

Proof. Define a sequence of functions on $\Omega$ by

$$
\bar{\phi}_{\epsilon}= \begin{cases}C+\frac{1}{C}\left(-\frac{1}{4 \pi(1+\alpha)} \log \left(1+g(0) \frac{\pi}{1+\alpha} \cdot \frac{|x|^{2(1+\alpha)}}{\epsilon^{2(1+\alpha)}}\right)+b\right), & x \in \bar{B}_{R \epsilon} \\ \frac{G-\eta \psi}{C}, & x \in B_{2 R \epsilon} \backslash \bar{B}_{R \epsilon} \\ \frac{G}{C^{\prime}} & x \in \Omega \backslash B_{2 R \epsilon}\end{cases}
$$


where $G$ is the Green function and $\psi$ is the regular part as $(3.28), R=(-\log \epsilon)^{\frac{1}{1+\alpha}}, \quad \eta \epsilon$ $C_{0}^{1}\left(B_{2 R \epsilon}\right)$ satisfying that $\eta=1$ on $B_{R \epsilon}$ and $|\nabla \eta| \leq \frac{2}{R \epsilon}, b$ and $C$ are constants depending only on $\epsilon$ to be determined later. Here and in the sequel, $B_{r}$ stands for a ball centered at 0 with radius $r$. Clearly $B_{2 R \epsilon} \subset \Omega$ provided that $\epsilon$ is sufficiently small. In order to assure that $\bar{\phi}_{\epsilon} \in W_{0}^{1,2}(\Omega)$, we set

$$
C+\frac{1}{C}\left(-\frac{1}{4 \pi(1+\alpha)} \log \left(1+\frac{g(0) \pi}{1+\alpha} R^{2(1+\alpha)}\right)+b\right)=\frac{1}{C}\left(-\frac{1}{2 \pi} \log (R \epsilon)+C_{G}\right),
$$

which gives

$$
C^{2}=-\frac{1}{2 \pi} \log \epsilon+C_{G}-b+\frac{1}{4 \pi(1+\alpha)} \log \frac{g(0) \pi}{1+\alpha}+O\left(\frac{1}{R^{2(1+\alpha)}}\right)
$$

Noting that $\psi(x)=O(|x|)$ as $x \rightarrow 0$, we have $|\nabla(\eta \psi)|=O(1)$ as $\epsilon \rightarrow 0$. It follows that

$$
\int_{B_{2 R e} \backslash B_{R \epsilon}}|\nabla(\eta \psi)|^{2} \mathrm{~d} x=O\left(R^{2} \epsilon^{2}\right), \quad \int_{B_{2 R \epsilon} \backslash B_{R \epsilon}} \nabla G \nabla(\eta \psi) \mathrm{d} x=O(R \epsilon) .
$$

Integration by parts gives

$$
\begin{aligned}
\int_{\Omega \backslash B_{R \epsilon}}|\nabla G|^{2} \mathrm{~d} x & =-\int_{\Omega \backslash B_{R \epsilon}} G \Delta G \mathrm{~d} x-\int_{\partial B_{R \epsilon}} G \frac{\partial G}{\partial \nu} \mathrm{d} s \\
& =-\frac{1}{2 \pi} \log (R \epsilon)+C_{G}+\beta \int_{\Omega} G^{2} \mathrm{~d} x+O(R \epsilon \log (R \epsilon)) .
\end{aligned}
$$

This leads to

$$
\begin{aligned}
\int_{\Omega \backslash B_{R \epsilon}}\left|\nabla \bar{\phi}_{\epsilon}\right|^{2} \mathrm{~d} x= & \frac{1}{C^{2}} \int_{\Omega \backslash B_{R \epsilon}}|\nabla G|^{2} \mathrm{~d} x+\frac{1}{C^{2}} \int_{B_{2 R \epsilon} \backslash B_{R \epsilon}}|\nabla(\eta \psi)|^{2} \mathrm{~d} x \\
& \quad-\frac{1}{C^{2}} \int_{B_{2 R \epsilon} \backslash B_{R e}} \nabla G \nabla(\eta \psi) \mathrm{d} x \\
= & \frac{1}{C^{2}}\left(-\frac{1}{2 \pi} \log (R \epsilon)+C_{G}+\beta \int_{\Omega} G^{2} \mathrm{~d} x+O(R \epsilon \log (R \epsilon))\right) .
\end{aligned}
$$

Also we have

$$
\begin{aligned}
\int_{B_{R e}}\left|\nabla \bar{\phi}_{\epsilon}\right|^{2} \mathrm{~d} x & =\frac{1}{4(1+\alpha)^{2} C^{2}} \int_{B_{R \epsilon}} \frac{g^{2}(0) \frac{|x|^{2+4 \alpha}}{\epsilon^{4+4 \alpha}}}{\left(1+g(0) \frac{\pi}{1+\alpha}\left|\frac{x}{\epsilon}\right|^{2(1+\alpha)}\right)^{2}} \mathrm{~d} x \\
& =\frac{1}{4(1+\alpha)^{2} C^{2}} \int_{B_{R}} \frac{g^{2}(0)|y|^{2+4 \alpha}}{\left(1+g(0) \frac{\pi}{1+\alpha}|y|^{2(1+\alpha)}\right)^{2}} \mathrm{~d} x \\
& =\frac{\pi}{2(1+\alpha)^{2} C^{2}} \int_{0}^{R} \frac{g^{2}(0) r^{3+4 \alpha}}{\left(1+g(0) \frac{\pi}{1+\alpha} r^{2(1+\alpha)}\right)^{2}} \mathrm{~d} r
\end{aligned}
$$




$$
\begin{aligned}
& =\frac{1}{4 \pi(1+\alpha) C^{2}} \int_{0}^{g(0) \frac{\pi}{1+\alpha} R^{2+2 \alpha}} \frac{t}{(1+t)^{2}} \mathrm{~d} t \\
& =\frac{1}{4 \pi(1+\alpha) C^{2}}\left(\log \frac{g(0) \pi}{1+\alpha}-1+\log R^{2+2 \alpha}+O\left(\frac{1}{R^{2+2 \alpha}}\right)\right) .
\end{aligned}
$$

Hence

$$
\begin{aligned}
\int_{\Omega}\left|\nabla \bar{\phi}_{\epsilon}\right|^{2} \mathrm{~d} x=\frac{1}{C^{2}}( & \left.-\frac{\log \epsilon}{2 \pi}+C_{G}+\beta \int_{\Omega} G^{2} \mathrm{~d} x-\frac{1}{4 \pi(1+\alpha)}+\frac{1}{4 \pi(1+\alpha)} \log \frac{g(0) \pi}{1+\alpha}\right) \\
& +O\left(\frac{1}{R^{2+2 \alpha}}\right)+O(R \epsilon \log (R \epsilon)) .
\end{aligned}
$$

Note that

$$
\int_{\Omega} \bar{\phi}_{\epsilon}^{2} \mathrm{~d} x=\frac{1}{C^{2}}\left(\int_{\Omega} G^{2} \mathrm{~d} x+O(R \epsilon)\right)
$$

and $O(R \epsilon \log (R \epsilon))=O\left(\frac{1}{R^{2+2 \alpha}}\right)$. If we choose the suitable constant $C$ such that

$$
\left\|\bar{\phi}_{\epsilon}\right\|_{1, \beta}=\int_{\Omega}\left|\nabla \bar{\phi}_{\epsilon}\right|^{2} \mathrm{~d} x-\beta \int_{\Omega} \bar{\phi}_{\epsilon}^{2} \mathrm{~d} x=1,
$$

the we must have

$$
C^{2}=-\frac{1}{2 \pi} \log \epsilon+C_{G}-\frac{1}{4 \pi(1+\alpha)}+\frac{1}{4 \pi(1+\alpha)} \log \frac{g(0) \pi}{1+\alpha}+O\left(\frac{1}{R^{2+2 \alpha}}\right) .
$$

Combine (4.10) and (4.11), we obtain

$$
b=\frac{1}{4 \pi(1+\alpha)}+O\left(\frac{1}{R^{2+2 \alpha}}\right)
$$

In view of (4.11) and (4.12), there holds on $B_{R \epsilon}$,

$$
\begin{gathered}
4 \pi(1+\alpha) \bar{\phi}_{\epsilon}^{2} \geq 4 \pi(1+\alpha) C^{2}-2 \log \left(1+g(0) \frac{\pi}{1+\alpha} \cdot \frac{|x|^{2(1+\alpha)}}{\epsilon^{2(1+\alpha)}}\right)+8 \pi(1+\alpha) b \\
=-2 \log \left(1+g(0) \frac{\pi}{1+\alpha} \cdot \frac{|x|^{2(1+\alpha)}}{\epsilon^{2(1+\alpha)}}\right)-2(1+\alpha) \log \epsilon+1 \\
+4 \pi(1+\alpha) C_{G}+\log \frac{g(0) \pi}{1+\alpha}+O\left(\frac{1}{R^{2+2 \alpha}}\right)
\end{gathered}
$$

which together with the estimate

$$
g(0) \int_{B_{R}} \frac{|y|^{2 \alpha}}{\left(1+g(0) \frac{\pi}{1+\alpha}|y|^{2+2 \alpha}\right)^{2}} \mathrm{~d} y=2 \pi g(0) \int_{0}^{R} \frac{r^{1+2 \alpha}}{\left(1+g(0) \frac{\pi}{1+\alpha} r^{2+2 \alpha}\right)^{2}} \mathrm{~d} r
$$




$$
=\int_{0}^{g(0) \frac{\pi}{1+\alpha} R^{2+2 \alpha}} \frac{1}{(1+t)^{2}} \mathrm{~d} t=1-\frac{1}{1+g(0) \frac{\pi}{1+\alpha} R^{2+2 \alpha}}
$$

leads to

$$
\begin{aligned}
& \int_{B_{R \epsilon}} g(x)|x|^{2 \alpha} e^{4 \pi(1+\alpha) \bar{\phi}_{\epsilon}^{2}} \mathrm{~d} x \\
\geq & \frac{g(0) \pi}{1+\alpha} \epsilon^{-2(1+\alpha)} e^{4 \pi(1+\alpha) C_{G}+1+O\left(\frac{1}{R^{2+2 \alpha}}\right)} \int_{B_{R \epsilon}} \frac{g(x)|x|^{2 \alpha}}{\left(1+g(0) \frac{\pi}{1+\alpha} \frac{|x|^{2+2 \alpha}}{\epsilon^{2+2 \alpha}}\right)^{2}} \mathrm{~d} x \\
= & \frac{g(0) \pi}{1+\alpha} e^{4 \pi(1+\alpha) C_{G}+1+O\left(\frac{1}{R^{2}+2 \alpha}\right)}\left(g(0)+o_{\epsilon}(1)\right) \int_{B_{R}} \frac{|y|^{2 \alpha}}{\left(1+g(0) \frac{\pi}{1+\alpha}|y|^{2+2 \alpha}\right)^{2}} \mathrm{~d} y \\
= & \frac{g(0) \pi}{1+\alpha} e^{4 \pi(1+\alpha) C_{G}+1+O\left(\frac{1}{R^{2}+2 \alpha}\right)}\left(1+o_{\epsilon}(1)\right)\left(1-\frac{1}{1+g(0) \frac{\pi}{1+\alpha} R^{2+2 \alpha}}\right) \\
= & \frac{g(0) \pi}{1+\alpha} e^{4 \pi(1+\alpha) C_{G}+1+O\left(\frac{1}{R^{2+2 \alpha}}\right)}+o_{\epsilon}(1)+O\left(\frac{1}{R^{2+2 \alpha}}\right) .
\end{aligned}
$$

On the other hand, since

$$
\begin{aligned}
& \int_{B_{2 R \epsilon}} g(x)|x|^{2 \alpha} \mathrm{d} x=O\left(\frac{1}{R^{2+2 \alpha}}\right), \\
& \int_{B_{2 R \epsilon}} g(x)|x|^{2 \alpha} G^{2} \mathrm{~d} x=O\left((R \epsilon)^{2+2 \alpha} \log ^{2}(R \epsilon)\right)=O\left(\frac{1}{R^{2+2 \alpha}}\right),
\end{aligned}
$$

we obtain

$$
\begin{aligned}
& \int_{\Omega \backslash B_{R \epsilon}} g(x)|x|^{2 \alpha} e^{4 \pi(1+\alpha) \bar{\phi}_{\epsilon}^{2}} \mathrm{~d} x \\
& \geq \int_{\Omega \backslash B_{2 R e}} g(x)|x|^{2 \alpha}\left(1+4 \pi(1+\alpha) \phi_{\epsilon}^{2}\right) \mathrm{d} x \\
& =\int_{\Omega \backslash B_{2 R \epsilon}} g(x)|x|^{2 \alpha} \mathrm{d} x+\frac{4 \pi(1+\alpha)}{C^{2}} \int_{\Omega \backslash B_{2 R \epsilon}} g(x)|x|^{2 \alpha} G^{2} \mathrm{~d} x \\
& =\int_{\Omega} g(x)|x|^{2 \alpha} \mathrm{d} x+\frac{4 \pi(1+\alpha)}{C^{2}} \int_{\Omega} g(x)|x|^{2 \alpha} G^{2} \mathrm{~d} x+O\left(\frac{1}{R^{2+2 \alpha}}\right) \text {. }
\end{aligned}
$$

Therefore

$$
\begin{gathered}
\int_{\Omega} g(x)|x|^{2 \alpha} e^{4 \pi(1+\alpha) \bar{\phi}_{\epsilon}^{2}} \mathrm{~d} x \\
\geq \int_{\Omega} g(x)|x|^{2 \alpha} \mathrm{d} x+\frac{4 \pi(1+\alpha)}{C^{2}} \int_{\Omega} g(x)|x|^{2 \alpha} G^{2} \mathrm{~d} x \\
\quad+\frac{g(0) \pi}{1+\alpha} e^{4 \pi(1+\alpha) C_{G}+1+O\left(\frac{1}{R^{2+2 \alpha}}\right)}+O\left(\frac{1}{R^{2+2 \alpha}}\right) .
\end{gathered}
$$


In view of $R=(-\log \epsilon)^{\frac{1}{1+\alpha}}$, we have $\frac{1}{R^{2+2 \alpha}}=o_{\epsilon}(1)$. And thus we obtain

$$
\int_{\Omega} V(x) e^{4 \pi(1+\alpha) \bar{\phi}_{\epsilon}^{2}} \mathrm{~d} x>\int_{\Omega} V(x) \mathrm{d} x+\frac{g(0) \pi}{1+\alpha} e^{4 \pi(1+\alpha) C_{G}+1}
$$

provided that $\epsilon>0$ is chosen sufficiently small.

\section{Acknowledgments}

The authors are supported partially by NSFC of China (No. 11271253 and No. 11771285). The authors also thank reviewers for their helpful comments on earlier draft of this paper.

\section{References}

[1] Iula S., Mancini G., Extremal Functions for Singular Moser-Trudinger Embeddings. Nonlinear Anal., 156 (2017), 215-248.

[2] Yang Y. Y., Zhu X. B., Blow-up analysis concerning singular Trudinger-Moser inequality in dimension two. J. Funct. Anal., 272 (2017), 3347-3374.

[3] Moser J., A sharp form of an inequality by N. Trudinger. Indiana Univ. Math. J., 20 (1971), 1077-1092.

[4] Pohozaev S., The sobolev embedding in the special case $p l=n$. Proceedings of the Technical Scientific Conference on Advances of Scientific Reseach. Mathmatic sections, Mosco. Energet. inst, (1965) 158-170.

[5] Trudinger N. S., On embeddings into Orlicz spaces and some applications. J. Math. Mech., 17 (1967), 473-483.

[6] Carleson L., Chang S-Y. A., On the existence of an extremal function for an inequality of J. Moser. Bull. Sci. Math., 110 (1986), 113-127.

[7] Flucher M., Extremal functions for the Trudinger-Moser inequality in two dimensions. Comment. Math. Helv., 67 (1992), 471-497.

[8] Adimurthi A., Tintarev C., On compactness in the Trudinger-Moser inequality. Ann. Sc. Norm. Super. Pisa Ci. Sci., 13 (2014), 399-416.

[9] Malchiodi A., Martinazzi L., Critical points of the Moser-Trudinger functional on a disk. J. Eur. Math. Soc., 16 (2014), 893-908.

[10] Mancini G., Sandeep K., Moser-Trudinger inequality on comformal discs. Commun. Contemp . Math., 12 (2010), 1055-1068.

[11] Adimurthi A., Sandeep K., A singular Moser-Trudinger embedding and its applications. Nolinear Differential Equations Appl., 13 (2007), 585-603.

[12] Csató G., Roy P., Extremal functions for the singular Moser-Trudinger inequality in 2 dimensions. Calc. Var. Partial Differential Equations, 54 (2015), 2341-2366.

[13] Tintarev C., Trudinger-Moser inequality with remainder terms. J. Funct. Anal., 266 (2014), 55-66.

[14] Adimurthi, Druet O., Blow-up analysis in dimension 2 and a sharp form of Trudinger-Moser inequality. Comm. partial Differential Equations, 29 (2004), 295-322.

[15] Lin K., Extremal functions for Moser's inequality. Trans. Amer. Math. Soc., 348 (1996), 26632671. 
[16] Lu G. Z., Yang Y. Y., Sharp constant and extremal function for the improved Moser-Trudinger inequality involving $L^{p}$ norm in two dimension. Discrete Contin. Dyn. Syst., 25 (2009), 963979.

[17] do Ò J. M., de Souza M., A sharp inequality of Trudinger-Moser type and extremal functions in $H^{1, n}\left(\mathbb{R}^{n}\right)$. J. Differential Equations, 258 (2015), 4062-4101.

[18] de Souza M., do Oे J. M., On a class of singular Trudinger-Moser type inequalities and its applications. Math. Nachr, 284 (2011), 1754-1776.

[19] Yang Y. Y., A sharp form of Moser-Trudinger inequality in high dimension. J. Functi. Anal., 239 (2006), 100-126.

[20] Yang Y. Y., Extremal functions for Trudinger-Moser inequalities of Adimurthi-Druet type in dimension two. J. Diffferential Equations, 258 (2015), 3161-3193.

[21] Yang Y. Y., Zhu X. B., An improved Hardy-Trudinger-Moser inequality. Ann. Global Ann. Geom., 49 (2016), 23-41.

[22] Zhu J. Y., Improved Moser-Trudinger inequality involving $L^{p}$ norm in $n$ dimensions. Adv. Nonlinear Stud., 14 (2014), 273C293.

[23] Chen W., Li C., Classification of solutions of some nonlinear elliptic equations. Duke Math. J., 63 (1991), 615-623.

[24] Prajapat J., Tarantello G., On a class of elliptic problems in $\mathbb{R}^{2}$ : symmetry and uniquess results. Proc. Roy. Soc., 131 (2001), 967-985.

[25] Lions P.-L., The concentration-compactness pricinple in the calculus of variations, Limit Case. I. Rev. Mat. Iberoamericana, 1 (1985), 145-201.

[26] Struwe M., Positive solutions of critical semilinear elliptic equations on non-contractible planar domain. J. Eur. Math. Soc., 2 (2000), 329-388.

[27] Wang G. F., Ye D., A hardy-Moser-Trudinger inequality. Adv. Math., 230 (2012), 294-320. 